OPEN ACCESS

Edited by:

Christophe Hirtz,

Université de Montpellier, France

Reviewed by:

Alino Martinez-Marcos,

University of Castilla La Mancha,

Spain

Daniel Saiz Sanchez, University of Castilla La Mancha,

Spain

Bruno P. Imbimbo,

Chiesi Farmaceutici, Italy

*Correspondence:

Enrique Santamaría esantamma@navarra.es

${ }^{\dagger}$ These authors share senior authorship

Received: 15 November 2018 Accepted: 23 May 2019

Published: 11 June 2019

Citation:

Lachen-Montes M González-Morales A, Palomino M, Ausin K, Gómez-Ochoa M,

Zelaya MV, Ferrer I,

Pérez-Mediavilla A,

Fernández-Irigoyen $\mathrm{J}$ and Santamaría E (2019) Early-Onset Molecular Derangements in the Olfactory Bulb of Tg2576 Mice: Novel Insights Into the Stress-Responsive Olfactory Kinase Dynamics in

Alzheimer's Disease. Front. Aging Neurosci. 11:141. doi: 10.3389/fnagi.2019.00141

\section{Early-Onset Molecular} Derangements in the Olfactory Bulb of Tg2576 Mice: Novel Insights Into the Stress-Responsive Olfactory Kinase Dynamics in Alzheimer's Disease

\author{
Mercedes Lachen-Montes ${ }^{1,2,3}$, Andrea González-Morales ${ }^{1,2,3}$, Maialen Palomino ${ }^{1}$, \\ Karina Ausin ${ }^{2,3}$, Marta Gómez-Ochoa ${ }^{4}$, María Victoria Zelaya ${ }^{1,3,4}$, Isidro Ferrer ${ }^{5}$, \\ Alberto Pérez-Mediavilla ${ }^{3,6}$, Joaquín Fernández-Irigoyen ${ }^{1,2,3 \dagger}$ \\ and Enrique Santamaría ${ }^{1,2,3 * \dagger}$
}

\begin{abstract}
${ }^{1}$ Clinical Neuroproteomics Group, NavarraBiomed, Complejo Hospitalario de Navarra (CHN), Universidad Pública de Navarra (UPNA), Pamplona, Spain, ${ }^{2}$ Proteored-ISCIII Proteomics Unit, NavarraBiomed, Complejo Hospitalario de Navarra (CHN), Universidad Pública de Navarra (UPNA), Pamplona, Spain, ${ }^{3}$ IDISNA, Navarra Institute for Health Research, Pamplona, Spain, ${ }^{4}$ Department of Pathology, Complejo Hospitalario de Navarra, Pamplona, Spain, ${ }^{5}$ nstitut de Neuropatologia, IDIBELL-Hospital Universitari de Bellvitge, Universitat de Barcelona, L'Hospitalet de Llobregat, Centro de Investigación Biomédica en Red de Enfermedades Neurodegenerativas (CIBERNED), Barcelona, Spain, ${ }^{6}$ Neurobiology of Alzheimer's Disease, Department of Biochemistry, Center for Applied Medical Research (CIMA), Neurosciences Division, University of Navarra, Pamplona, Spain
\end{abstract}

The olfactory bulb $(\mathrm{OB})$ is the first processing station in the olfactory pathway. Despite smell impairment, which is considered an early event in Alzheimer's disease (AD), little is known about the initial molecular disturbances that accompany the AD development at olfactory level. We have interrogated the time-dependent $\mathrm{OB}$ molecular landscape in Tg2576 AD mice prior to the appearance of neuropathological amyloid plaques (2-, and 6-month-old), using combinatorial omics analysis. The metabolic modulation induced by overproduction of human mutated amyloid precursor protein (APP) clearly differs between both time points. Besides the progressive perturbation of the APP interactome, functional network analysis unveiled an inverse regulation of downstream extracellular signal-regulated kinase (ERK1/2), and p38 mitogen-activated protein kinase (MAPK) routes in 2-month-old Tg2576 mice with respect to wild-type (WT) mice. In contrast, Akt and MAPK kinase 4 (SEK1)/ stress-activated protein kinase (SAPK) axis were parallel activated in the OB of 6-months-old-Tg2576 mice. Furthermore, a survival kinome profiling performed during the aging process (2-, 6-, and 18-month-old) revealed that olfactory APP overexpression leads to changes in the activation dynamics of protein kinase $A(P K A)$,

\footnotetext{
Abbreviations: AD, Alzheimer's disease; Akt, Protein kinase B; APP, Amyloid precursor protein; ATF2, Activating Transcription Factor 2; CaMKII, $\mathrm{Ca}^{2+} /$ calmodulin-dependent protein kinase II; ERK, Extracellular signal-regulated kinase; FAK, Focal adhesion kinase; MEK, ERK Activator Kinase; OB, Olfactory bulb; p38 MAPK, p38 mitogen-activated protein kinase; PDK1, Phosphoinositide-dependent protein kinase 1; Phb, Prohibitin; PKA, protein kinase A; PKC, protein kinase C; PP5, Serine/threonine-protein phosphatase 5; SAPK/JNK, stress-activated protein kinase/Jun-amino terminal kinase; SEK1/MKK4, Mitogen-activated protein kinase Kinase 4.
} 
and SEK1/MKK4-SAPK/JNK between 6 and 18 months of age, when memory deficits appear and $A D$ pathology is well established in transgenic mice. Interestingly, both olfactory pathways were differentially activated in a stage-dependent manner in human sporadic AD subjects with different neuropathological grading. Taken together, our data reflect the early impact of mutated APP on the OB molecular homeostasis, highlighting the progressive modulation of specific signaling pathways during the olfactory amyloidogenic pathology.

Keywords: Alzheimer's disease, olfactory bulb, network biology, proteomics, transcriptomics, Tg2576 mice

\section{INTRODUCTION}

Together with typical symptoms, such as memory loss and behavioral disorders, Alzheimer's disease (AD) patients present olfactory dysfunction in $90 \%$ of the cases (Attems et al., 2014; Daulatzai, 2015). However, the etiology of this smell impairment is complex and remains mostly unknown. Neuropathological studies support the hypothesis that hyperphosphorylated Tau and $A \beta$ aggregation, present in the olfactory bulb $(\mathrm{OB})$, are early and important events in the AD pathophysiology (Rey et al., 2018). It has been demonstrated that these protein deposits reflect the presence and severity of $\mathrm{AD}$ pathology in other brain structures (Attems et al., 2014).

Although no animal model recapitulates the entirety of human AD pathology (Sasaguri et al., 2017), some AD transgenic mouse models also present olfactory deficits. Tg2576 transgenic mice express an isoform of human amyloid precursor protein (APP) with double mutation K670N, M671L (hAPPSw; Hsiao et al., 1996). Production of $A \beta 40$ and $A \beta 42$ and plaques formation are observed in cortical and hippocampal areas of these mice at the age of 11-13 months (Puzzo et al., 2015). Previous reports have pointed out that the accumulation of $\mathrm{A} \beta$ peptide is related to age-related memory decline in these mice (Chen et al., 2000; Janus et al., 2000; Westerman et al., 2002), inducing synaptic deficits and mitochondrial imbalance (Reddy et al., 2004; Jacobsen et al., 2006). The presence of APP processing products has been characterized in the OB of 1-month-old Tg2576 mice (Lehman et al., 2003). This progressive $A \beta$ deposition in specific olfactory structures is accompanied by behavioral deficits in odor habituation and discrimination, diminished rate of $\mathrm{OB}$ neurogenesis, and an altered volume of the $\mathrm{OB}$ granular cell layer in $\mathrm{Tg} 2576$ mice (Guérin et al., 2009; Young et al., 2009; Wesson et al., 2010, 2011).

Several studies using human $\mathrm{AD}$ brains and $\mathrm{AD}$ mouse models have demonstrated that the deposition of amyloid plaques is accompanied by an alteration in the OB's molecular homeostasis (Zelaya et al., 2015; Lachén-Montes et al., 2016, 2017; Palomino-Alonso et al., 2017). However, it is still unclear how the progressive amyloidogenic pathology affects the $\mathrm{OB}$ functionality in the absence of plaques. In this study, we have used two high-throughput technological platforms, combining transcriptomic and proteomic approaches to ascertain the magnitude and chronology of the $\mathrm{OB}$ molecular remodeling in Tg2576 mice at two stages of AD: long before (2 months of age), and immediately before (6 months of age) the appearance of $A \beta$ plaques, with respect to age-matched background strain wild-type (WT) mice. Our workflow has revealed stagedependent molecular pathways and kinase activation dynamics that are disturbed during the initial phase of the amyloid pathology, providing basic information for understanding how olfactory molecular networks evolve as the $\mathrm{AD}$ pathology progresses at early stages.

\section{MATERIALS AND METHODS}

\section{Human Samples}

According to the Spanish Law 14/2007 of Biomedical Research, informed written consent forms of Brain Bank of IDIBELL, and Neurological Tissue Bank of IDIBAPS-Hospital Clinic (Barcelona, Spain) was obtained for research purposes from relatives of patients included in this study. The study was conducted in accordance with the Declaration of Helsinki and all assessments, post-mortem evaluations, and procedures were previously approved by the Clinical Ethics Committee of Navarra Health Service. For the validation phase, $14 \mathrm{AD}$ cases were distributed into different groups according to specific consensus diagnostic criteria (Braak et al., 2006; Alafuzoff et al., 2008): initial (Braak I-II), intermediate (Braak III-IV), and advanced $\mathrm{AD}$ stages (Braak V-VI; $n=4-5 /$ group). Five cases from elderly subjects with no history or histological findings of any neurological disease were used as a control group. Eighty-five percentage of human brains considered in this study had a post-mortem interval (PMI) lower than $15 \mathrm{~h}$ (Table 1).

\section{Materials}

The following reagents and materials were used. Electrophoresis reagents were purchased from Bio-rad and Trypsin from Promega. Antibodies used during the study are summarized in Table 2.

\section{Animals}

Transgenic mice (Tg2576) overexpressing hAPP, carrying the Swedish (K670N/M671L) familial AD mutation and under control of the prion promoter (Hsiao et al., 1996), were used. Mice were on an inbred C57BL/6/SJL genetic background. The animals were maintained in positive pressureventilated racks at $25 \pm 1{ }^{\circ} \mathrm{C}$ with a $12 \mathrm{~h}$ light/dark cycle, fed ad libitum with a standard rodent pellet diet (Global Diet 2014; Harlan Laboratories, Indianapolis, IN, USA) and had free access to filtered and UV-irradiated 
TABLE 1 | General characteristics of the subjects included in this study.

\begin{tabular}{|c|c|c|c|c|}
\hline Case & Sex & Age & PMI (hours) & Pathological diagnosis \\
\hline \multicolumn{5}{|l|}{ Controls } \\
\hline BK-0300 & $\mathrm{F}$ & 75 & 20 & ARP I-II \\
\hline BK-1378 & M & 78 & 6 & vascular encephalopathy \\
\hline BK-1078 & $\mathrm{F}$ & 84 & 6 & vascular encephalopathy, NFT I \\
\hline BK-1195 & $\mathrm{F}$ & 82 & 8 & acute ictus, cerebellar hematoma \\
\hline BK-1563 & M & 79 & 15 & acute ictus, AgD II \\
\hline \multicolumn{5}{|l|}{ initial AD } \\
\hline $\mathrm{A} 13 / 70$ & $\mathrm{~F}$ & 79 & 10 & $A D \| / A$ \\
\hline A14/29 & $\mathrm{F}$ & 78 & 3.5 & $A D \| / A$ \\
\hline A14/33 & $\mathrm{M}$ & 62 & 9.5 & AD $\| / 0$ \\
\hline $\mathrm{A} 14 / 52$ & M & 70 & 3 & AD ॥/O \\
\hline \multicolumn{5}{|c|}{ intermediate AD } \\
\hline A12/42 & $\mathrm{F}$ & 82 & 17 & $A D I V / A$ \\
\hline A12/47 & M & 81 & 5 & AD III/A \\
\hline A12/48 & M & 84 & 12 & AD IV/A \\
\hline A12/54 & M & 89 & 3 & $A D I V / A$ \\
\hline A15/17 & M & 84 & 20 & AD III/A \\
\hline \multicolumn{5}{|c|}{ Advanced AD } \\
\hline CS-1445 & $\mathrm{F}$ & 73 & 3.5 & $\mathrm{AD} \mathrm{VI/C}+$ moderate CAA \\
\hline CS-0662 & $M$ & 75 & 4 & $A D V I / C$ \\
\hline CS-0535 & $\mathrm{F}$ & 81 & 4.5 & AD VI/C \\
\hline CS-0673 & M & 75 & 4.25 & $A D V I / C$ \\
\hline CS-1232 & M & 84 & 5 & $A D$ VI C + CAA \\
\hline
\end{tabular}

PMI, post-mortem interval; AD/ARP Alzheimer's disease related pathology; NFT: neurofibrillary tangles I-VI according to Braak, amyloid plaques A-C according to CERAD; CAA: cerebral amyloid angiopathy; AgD: argyrophilic grain disease.

TABLE 2 | Antibody used in this study.

\begin{tabular}{lcc}
\hline Antibodies & Product number & Distributor \\
\hline anti-APP & 2450 & Cell signaling \\
anti-MEK1/2 & 9126 & \\
anti-phospho-MEK1/2 (S217/221) & 9154 & \\
anti-ERK1/2 & 9102 & \\
anti-phospho-ERK1/2 (T202/y204) & 4370 & \\
anti-Akt & 4685 & \\
anti-phospho-Akt (S473) & 4060 & \\
anti-p38 MAPK & 9212 & \\
anti-phospho-p38 MAPK (T180/Y182) & 9211 & \\
anti-phospho-ATF2 (T71) & 5112 & \\
anti-SAPK/JNK & 9252 & \\
anti-phospho-SAPK/JNK (T183/Y185) & 9255 & \\
anti-SEK1 & 9152 & \\
anti-phospho-SEK1 (S257/T261) & 9156 & \\
anti-PKA C-alpha & 4782 & \\
anti-phospho-PKA C (T197) & 5661 & \\
anti-PP5 & 2289 & \\
anti-PDK1 & 3062 & \\
anti-phospho-PDK1 (S241) & 3061 & \\
anti-phospho-PKC pan (T514) & 9379 & \\
anti-phospho-FAK (Y576/577) & 3281 & \\
anti-Phb1 & 2426 & \\
and anti-Phb2 & SAB4502356 & \\
Anti-PKC-pan & & \\
\hline
\end{tabular}

water. All animal care and experimental procedures were in accordance with European and Spanish regulations (86/609/CEE; RD1201/2005) and were approved by the Ethical Committee of the University of Navarra (no. 018/05). Twenty-four animals, divided into two sets, were used for proteomics and transcriptomics analysis (12 mice/approach), with at least three WT and three Tg2576 transgenic mice per stage (2- and 6-month-old). Additionally, 14- and 18-month-old animals were used for immunohistochemistry and cell survival routes signaling analysis. Table 3 summarizes the number and the purpose for each of the animals. The progressive development of $\mathrm{AD}$ signs in our colony has been previously described (CuadradoTejedor and García-Osta, 2014). We have previously observed that behavior (Morris Water Maze test, MWM) is completely normal and amyloid levels are equal to wild type at 2 months of age. At 6 months of age, mice show impaired cognitive functions in the contextual fear conditioning test, coinciding with the increased cortical and hippocampal soluble $\beta$ amyloid (A $\beta$ ) levels. At 12 months, the impairment in MWM is present in most of the mice, but few are normal and with less plaques (but they are present); and finally, in aged mice (17-18 months), the pathology is robust and $100 \%$ of mice shows plaques and MWM impairment.

\section{Sample Preparation for Proteomic Analysis}

Murine OB specimens were homogenized in lysis buffer containing $7 \mathrm{M}$ urea, $2 \mathrm{M}$ thiourea, $50 \mathrm{mM}$ DTT. The homogenates were spinned down at $100,000 \times \mathrm{g}$ for $1 \mathrm{~h}$ at $15^{\circ} \mathrm{C}$. Protein concentration was measured in the supernatants with the Bradford assay kit (Bio-Rad).

\section{Olfactory Bulb Proteomics}

Sample preparation, Protein Digestion and Peptide iTRAQ Labeling were performed as previously described (Zelaya et al., 2015; Lachén-Montes et al., 2017). Briefly, each tryptic digest was labeled according to the manufacturer's instructions with one isobaric amine-reactive tags as follows: set 1 (experiment with 2-months-old mice): Tag113, WT-1; Tag114, WT-2; Tag115, WT-3; Tag116, Tg2576-1; Tag117, Tg2576-2; Tag118, Tg2576-3, and Set 2 (experiment with 6months-old mice): Tag113, WT-1; Tag114, WT-2; Tag115, WT3; Tag116, Tg2576-1; Tag117, Tg2576-2; Tag118, Tg2576-3. After $2 \mathrm{~h}$ of incubation, the set of labeled samples were pooled and evaporated in a vacuum centrifuge. To increase the proteome coverage, the peptide pool was submitted to cation exchange chromatography using spin Columns (Pierce). Twelve fractions were collected (from $5 \mathrm{mM}$ to $250 \mathrm{mM}$ $\mathrm{KCl}$ ), concentrated using $\mathrm{C} 18$ zip tip solid phase extraction (Millipore), evaporated under vacuum and reconstituted into $10 \mu \mathrm{l}$ of $2 \%$ acetonitrile, $0.1 \%$ formic acid, $98 \%$ MilliQ-H20 prior to mass spectrometric analysis. Peptide mixtures were separated by reverse phase chromatography and analyzed by mass-spectrometry as previously described (PalominoAlonso et al., 2017). The raw MS/MS spectra search were processed using the MaxQuant software (v.1.5.8.3; Tyanova et al., 2016a) and searched against the Uniprot proteome reference for Mus musculus (Proteome ID: UP000000589, May 2017). The parameters used were as follows: initial maximum precursor (25 ppm), fragment mass deviations (40 ppm); variable modification (methionine oxidation and $\mathrm{N}$-terminal acetylation) and fixed modification (MMTS); enzyme (trypsin) with a maximum of one missed cleavages; minimum peptide length (seven amino acids); false discovery rate (FDR) for PSM and protein identification (1\%). Frequently observed 
TABLE 3 | Number of animals used in each experimental approach.

\begin{tabular}{|c|c|c|c|c|}
\hline Mice & Proteomics & Transcriptomics & Ab burden analysis & Signaling routes study \\
\hline 3 month-old wild type & 3 & 3 & 3 & 3 \\
\hline 3 month-old Tg2576 & 3 & 3 & 3 & 3 \\
\hline 6 month-old wild type & 3 & 3 & 3 & 3 \\
\hline 6 month-old Tg2576 & 3 & 3 & 3 & 3 \\
\hline 14 month-old wild type & - & - & 3 & - \\
\hline 14 month-old Tg2576 & - & - & 3 & - \\
\hline 18 month-old wild type & - & - & - & 3 \\
\hline 18 month-old Tg2576 & - & - & - & 3 \\
\hline
\end{tabular}

laboratory contaminants were removed. Protein identification was considered valid with at least one unique or "razor" peptide. The protein quantification was calculated using at least two razor + unique peptides, and statistical significance was calculated by a two-way Student $t$-test $(p<0.05)$. A 1.3 -fold change cut-off was used. Proteins with iTRAQ ratios below the low range (0.77) were considered to be down-regulated, whereas those above the high range (1.3) were considered to be upregulated. The Perseus software (version 1.5.6.0; Tyanova et al., 2016b) was used for statistical analysis and data visualization. Search results files and MS raw data were deposited to the ProteomeXchange Consortium ${ }^{1}$ via the PRIDE partner repository (Vizcaíno et al., 2014) with the dataset identifiers PXD007813 (username: reviewer31643@ebi.ac.uk; password: gMcgfWzD).

\section{Olfactory Bulb Transcriptomics}

OB Transcriptomics-Maxwell ${ }^{\circledR} 16$ simplyRNA Kit (Promega) was used to extract the OB mRNAs from Tg2576 mice and WT littermates. The sense cDNA was fragmented and biotinylated using the Affymetrix Clarion S Pico assay (902932). Affymetrix mouse Clarion $S$ chips were used according to the manufacturer protocols. Hybridization, washing, staining, scanning, and data analysis (Irizarry et al., 2003) were performed as previously described (Lachen-Montes et al., 2017). As in other transcriptomic studies performed in $\mathrm{AD}$ brains (Silva et al., 2012; Cuadrado-Tejedor et al., 2016), we worked with a $p$-value $<0.01$ (without using any method for multiple testing correction). Microarray data files were submitted to the GEO (Gene Expression Omnibus) database and are available under accession number GSE106643.

\section{Bioinformatics}

The identification of specifically dysregulated regulatory/metabolic networks in Tg2576, OBs was analyzed using QIAGEN's Ingenuity ${ }^{\circledR}$ Pathway Analysis (IPA; QIAGEN Redwood $\left.\mathrm{City}^{2}\right)$. The software generates significance values ( $p$-values) between each biological or molecular event and the imported molecules based on the Fisher's exact test $(p \leq 0.05)$. The IPA comparison analysis considers the signaling pathway rank according to the calculated $p$-value and reports it hierarchically.

\footnotetext{
${ }^{1}$ http://proteomecentral.proteomexchange.org

${ }^{2}$ www.qiagen.com/ingenuity
}

\section{Immunoblotting Analysis}

Equal amounts of OB protein $(5 \mu \mathrm{g})$ were resolved in $4 \%-15 \%$ TGX stain-Free gels (Bio-Rad). OB proteins derived from murine and human samples were electrophoretically transferred onto nitrocellulose membranes using a Trans-blot Turbo transfer system (up to 25V, 7 min; Bio-Rad). Equal loading of the gels was assessed by stain free digitalization and by Ponceau staining. Western-blotting was performed as previously described (Lachén-Montes et al., 2017). After densitometric analyses (Image Lab Software Version 5.2; Bio-Rad), optical density values were expressed as arbitrary units and normalized to total stain in each gel lane.

\section{Immunohistochemistry}

Under xylazine/ketamine anesthesia, animals were perfused transcardially with saline for $3 \mathrm{~min}$ at a $11 \mathrm{ml} / \mathrm{min}$ flow, and $4 \%$ paraformaldehyde in phosphate buffered saline (PBS) for $2 \mathrm{~min}$ at a $9 \mathrm{ml} / \mathrm{min}$ flow. After perfusion, brains were removed, post-fixed in $4 \%$ paraformaldehyde for $1 \mathrm{~h}$ at room temperature and cryoprotected in 30\% sucrose solution in PBS overnight at $4^{\circ} \mathrm{C}$. Brains were sliced into $40-\mu \mathrm{m}$-thick coronal sections along the rostral axis with a freezing microtome (Leica, Germany) and collected in 0.125 M PBS containing 2\% dimethylsulphoxide (Sigma), $20 \%$ glycerin (Panreac) and $0.05 \%$ sodium azide, and stored at $-20^{\circ} \mathrm{C}$ until their subsequent analysis. Five free-floating tissue sections, comprising the $\mathrm{OB}$ of four animals per age group (2-, 6- and 14-month old), were processed for immunohistochemistry. The sections were washed $(3 \times 10 \mathrm{~min})$ with a solution buffer containing PBS $0.125 \mathrm{M}(\mathrm{pH} 7.4)$, $0.5 \%$ Triton X-100 and $0.1 \%$ BSA. After washing, sections were treated with methanol and $\mathrm{H}_{2} \mathrm{O}_{2}$ to inhibit endogenous peroxidase activity and incubated in $70 \%$ formic acid for $5 \mathrm{~min}$ to expose the epitope. Subsequently, the sections were incubated overnight with a primary mouse antibody (6E10) raised against human $A \beta$ (amino acids 1-16; BioLegend, San Diego, CA, USA) diluted $1: 1,000$ in PBS $0.125 \mathrm{M}(\mathrm{pH}$ 7.4), $0.5 \%$ Triton $\mathrm{X}-100,0.1 \% \mathrm{BSA}$ and $5 \%$ normal goat sera. After washing $(3 \times 5 \mathrm{~min})$ in $\mathrm{PBS}$, sections were incubated for $30 \mathrm{~min}$ with biotinylated goat anti-mouse secondary antibody (DakoCytomation, Glostrup, Denmark) diluted 1:500 in PBS. The sections were then processed using the avidin-biotin-peroxidase complex (Vectastain kit, Vector Laboratories, Burlingame, CA, USA) and reacted with $0.05 \% 3,3^{\prime}$-diaminobenzidine tetrahydrochloride (DAB) and $0.015 \% \mathrm{H}_{2} \mathrm{O}_{2}$ in $50 \mathrm{mM}$ Tris $\mathrm{HCl}, \mathrm{pH}$ 7.2. After washing 
in deionized water, sections were mounted on gelatinized slides, counterstained with Thionine at $60^{\circ} \mathrm{C}$ (Panreac Quimica, Barcelona, Spain) and cover-slipped with DPX (VWR, Dublin, Ireland). With respect to human $\mathrm{OB}$ samples, formalinfixed, paraffin-embedded tissue sections from $\mathrm{OB}$ (derived from controls and $\mathrm{AD}$ cases) were sectioned at $5 \mu \mathrm{m}$ and counterstained with hemeatoxylin for immnuhistochemistry analysis with anti-SEK1 (ref. 9152; 1:50), anti-phospho-SEK1 (S257/T261; Ref. 9156; 1:250), anti-protein kinase A (PKA) C-alpha (ref. 4782; 1:50), anti-phospho-PKA C (T197; ref. 5661; 1:250). Visualization was performed by an automated slide immunostainer (Leica Bond Max) with BondPolymer Refine Detection (Leica Biosystems Newcastle Ltd., Newcastle upon Tyne, UK).

\section{RESULTS}

\section{APP Overproduction Induces Early, and Time-Dependent Molecular Derangements in the Olfactory Bulb of Tg2576 Mice}

First, we analyzed the olfactory A $\beta$ pathology in TG2576 mice. As shown in Figure 1, intraneuronal $A \beta$ immunoreactivity was observed in 2-month-old transgenic mice, detecting $\mathrm{A} \beta$ deposition in the form of diffuse plaques and mature plaques at the age of 6 and 14 months, respectively (see Supplementary Figure S1 for more details). As the primary aim of our study was to analyze early-onset molecular derangements in the $\mathrm{OB}$ of Tg2576 mice, we deeply monitored $\mathrm{OB}$ molecular disturbances at two time-points (2 and 6 months), using high-throughput molecular technologies (Figure 2A). At both time-points, Tg2576 mice displayed abundant full-length human APP expression in the OB (Figure 2B). To examine the consequences of initial incremental accumulation of APP on OB molecular homeostasis, we applied proteomics and transcriptomics with the final goal to decipher novel information about the $\mathrm{OB}$ site-specific molecular signature at early AD stages in 2-month and 6-month-old Tg2576 mice. To analyze the potential differences in olfactory molecular expression profiles, $\mathrm{OB}$ specimens for each experimental group (Tg2576 and WT mice) were subjected into chemical tags (iTRAQ) coupled to tandem mass spectrometry and into the RNA microarray platform. With respect to transcriptome-wide analysis, 187 protein-coding genes were differentially regulated in the $\mathrm{OB}$ of 2-month-old $\operatorname{Tg} 2576$ mice (46 down- and 141 up-regulated genes with respect to WT mice), whereas 287 differentially expressed genes were found in 6-monthold Tg2576 OBs (107 down- and 180 up-regulated genes with respect to WT mice; Figure 2B, and Supplementary Table S1). In the proteomic phase, 1,605 and 1,752 proteins were quantified at 2 and 6 months, respectively. The expression levels of 31 proteins were found to be significantly different between 2-month-old WT and Tg2576 mice (12 down- and 19 up-regulated proteins with respect to WT mice), and 61 differentially expressed proteins were detected at 6 months of age (26 down- and 35 up-regulated proteins with respect to WT animals; Figures 2B,C, and Supplementary Table S2).
To partially validate our quantitative LC-MS/MS approach, the increment in Serine/threonine-protein phosphatase 5 (PP5), a phosphatase that protects neurons against $\mathrm{A} \beta$ toxicity (Sanchez-Ortiz et al., 2009), was verified by Western-blotting in 6-month-old Tg2576 OB (Supplementary Figure S2A). Interestingly, the genes and proteins affected between both stages varied widely, with only one protein (APP) and nine genes common to the two time-points (Figure 2D). Of these nine genes, four were co-downregulated (FOS, ARC, NPAS4, RGSL1) and five were co-upregulated (STMN4, STMN2, F3, HIF3a, EMB).

\section{Functional Modules Progressively Disrupted by Human Mutated APP in the Olfactory Bulb of Tg2576 Mice}

To characterize in detail the proteogenomic modulation induced by the presence of human mutated APP at pre-plaque stages in the $\mathrm{OB}$, differential proteomic and transcriptomic datasets were merged, and functionally analyzed across specific biological functions (see Supplementary Table S3). Functional bioinformatic analysis revealed that the accumulation of APP resulted in disturbances of statistically over-represented molecular processes directly relevant to adhesion ( $p$-val: $0.0001)$, viability ( $p$-val: 0.01 ), and interaction of neuroglia ( $p$-val: 0.0001$)$, quantity ( $p$-val: 0.003$)$, development ( $p$-val: $0.04)$, and plasticity of synapse ( $p$-val: 0.001$)$, long-term potentiation ( $p$-val: 0.006$)$, growth of neurites ( $p$-val: 0.03 ), and neuronal proliferation ( $p$-val: 0.04$)$ in 2-month-old Tg2576 mice (Figure 3). In 6-month-old Tg2576 mice, the olfactory amyloid pathology predominantly resulted in the significant alteration of microtubule dynamics ( $p$-val: $0.0001)$, neuritogenesis ( $p$-val: 0.0005$)$, axonogenesis ( $p$-val: $0.007)$, synaptic transmission ( $p$-val: 0.01$)$, transmembrane potential of mitochondria ( $p$-val: 0.00001$)$, metabolism of ROS ( $p$-val: 0.02 ), and neurodegeneration of sensory neurons ( $p$-val: 0.02 ) between others (Figure 3). However, functional commonalities focused on synthesis, and concentration of lipids/fatty acids, cell death, astrocytosis, and accumulation of vesicles were also detected in 2- and 6-month-old Tg2576 OBs (Figure 3, and Supplementary Table S3). Considering that the discovery of unexpected connections between seemingly unrelated molecules and human mutated APP is a straightforward approach for the identification of novel $\mathrm{AD}$ causative targets involved in early olfactory neurodegeneration, we explored whether highly expressed APP isoform was potentially interconnected with differential molecular mediators identified in our proteogenomic approach. For that, functional interactomes were generated using IPA software. Interestingly, 35 differential functional interactors for APP were identified in the OB of Tg2576 mice at pre-plaque stages, suggesting the involvement in related biological functions (Figure 4). Specifically, at 2 months of age, olfactory APP is central to an interconnected molecular network composed of targets with specific subcellular distribution: (i) IGF2, OGN, TIMP3 at extracellular level; (ii) LRPAP1, NNAT, STMN2 in the plasma membrane; (iii) cytoplasmic GSTM3, MT-ND3, 

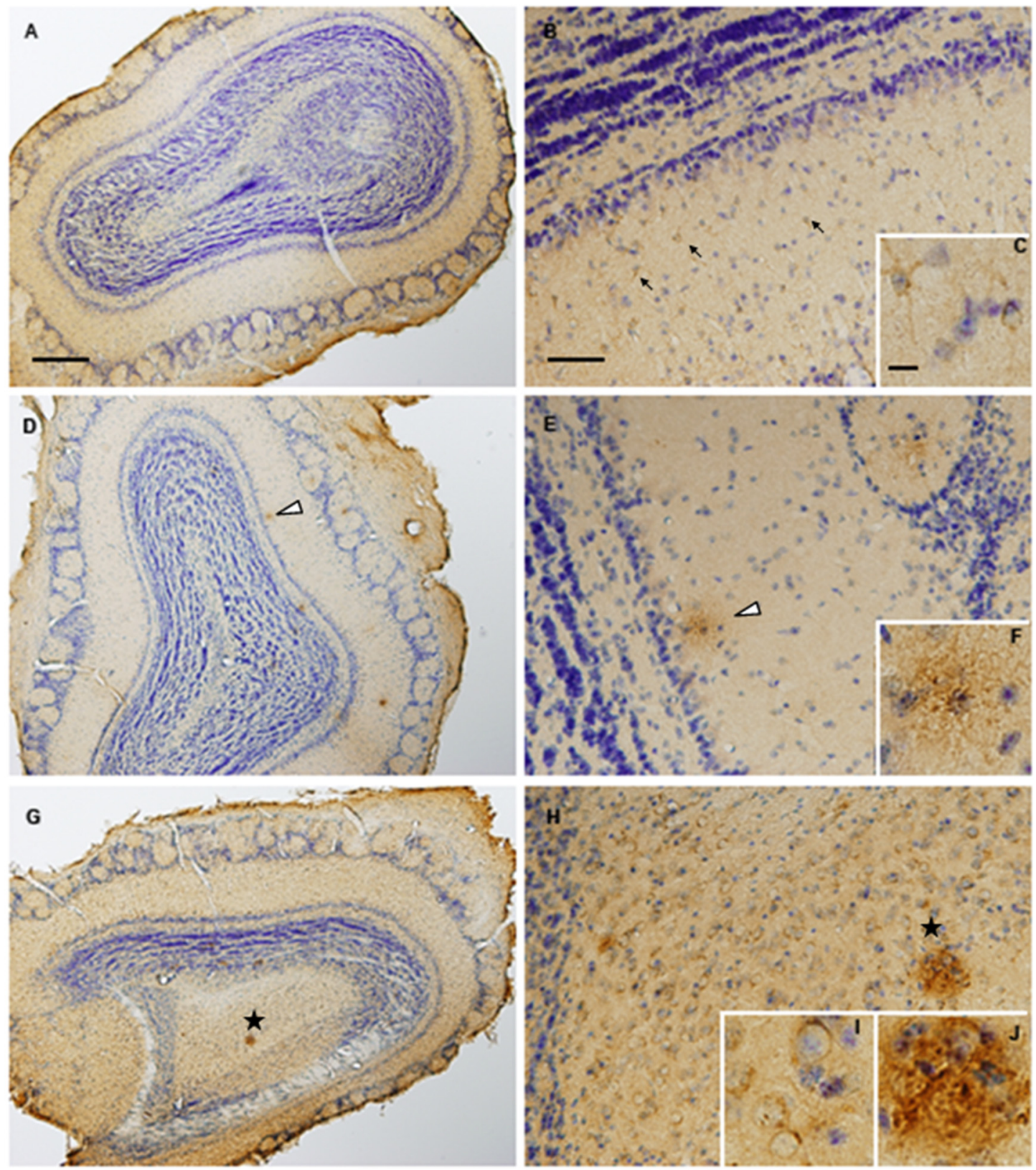

FIGURE 1 | Olfactory $\beta$-Amyloid A $\beta$ pathology increases with the disease progression in TG2576 mice. Olfactory bulbs (OBs) were harvested from 2-(A-C), 6-(D-F) and 14-(G-J) month-old Tg2576 mice. Intraneuronal A $\beta$ immunoreactivity can be observed in 2-month-old mice (arrow heads; panel B and more detailed in panel C). OB samples from 6-month-old animals (panels D-F) shows moderate A $\beta$ deposition in form of diffuse plaques (asterisk). By contrast, mature plaques (asterisk in panels $\mathbf{G}, \mathbf{H}$ and insert $\mathbf{J}$ ) and vascular A $\beta$ (insert I) is evident in 14-month-old Tg2576. Scale bars (500 $\mu \mathrm{m}$ for panels $\mathbf{A}, \mathbf{D}, \mathbf{G}, 100 \mu \mathrm{m}$ for panels $\mathbf{B}, \mathbf{E}, \mathbf{H})$ or $10 \mu \mathrm{m}(\mathbf{C}, \mathbf{F}, \mathbf{I}, \mathbf{J})$.

MAPK10, ACTG1, PRDX6, RNF24, NDUFA8, ARC, and S100B; and (iv) MECP2, FOSB, EGR1, FOS, EGR2 in the nucleus (Figure 4A). At 6 months of age, the olfactory APP interactome completely varied at extracellular (MBP, and CSF1), plasma membrane (GNG2, GAP43, STMN2, MERTK), cytoplasmic (APBA1, PVALB, MAPK3, GSR, CRYM, MAP2K4, $\mathrm{ABCD} 3, \mathrm{KLC} 2, \mathrm{FBXO} 2, \mathrm{ARC}$ ), and nuclear (FOS, TOP2B) levels (Figure 4B).
Additional integrative networks unveiled an early disruption of EIF2 signaling based on the up-regulation of a subset of ribosomal proteins (Figure 5A), suggesting that olfactory protein synthesis is compromised in 2-month-old Tg2576 mice. Furthermore, a dysregulation of specific subunits, corresponding to the mitochondrial complexes I and VI, was evidenced in the OB of 2-month-old Tg2576 mice, suggesting an olfactory mitochondrial impairment (Figure 5B). It is well-known 
A

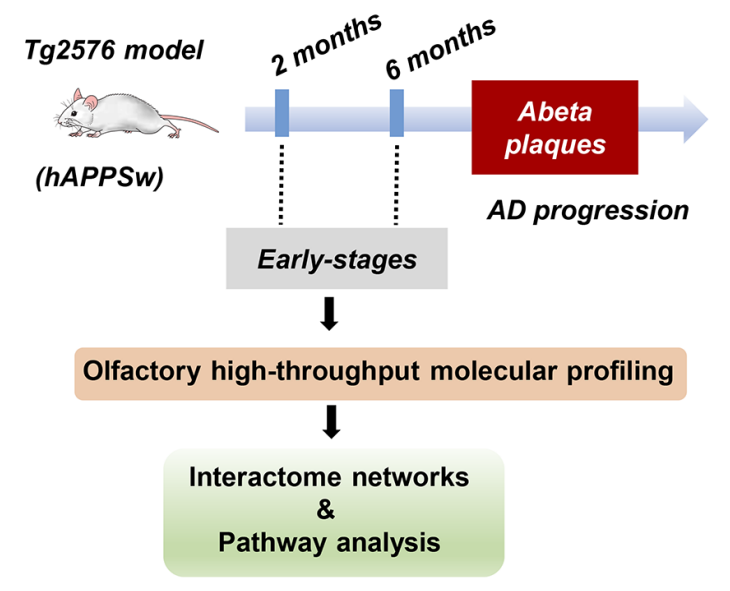

C

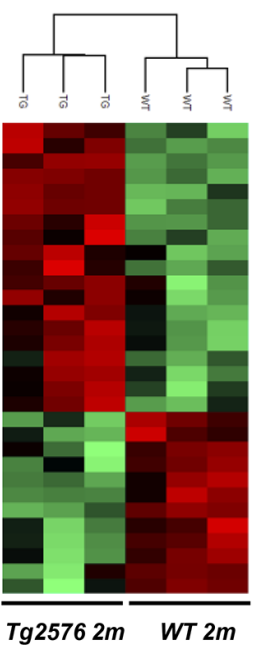

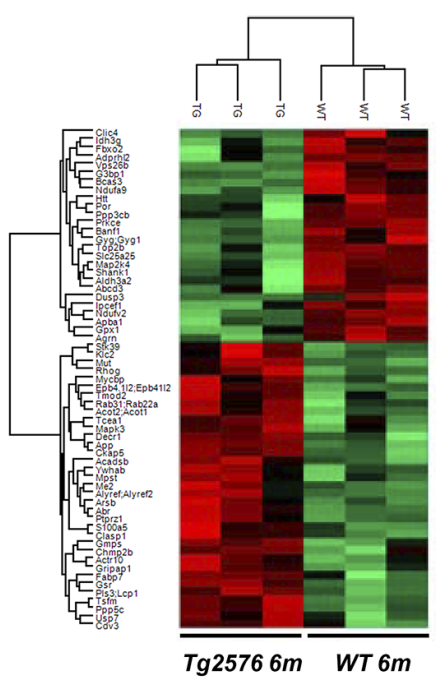

B
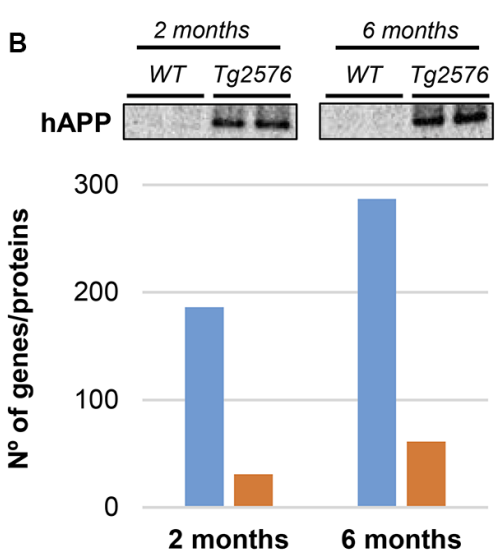

OB transcriptome $\quad \mathrm{OB}$ proteome

D

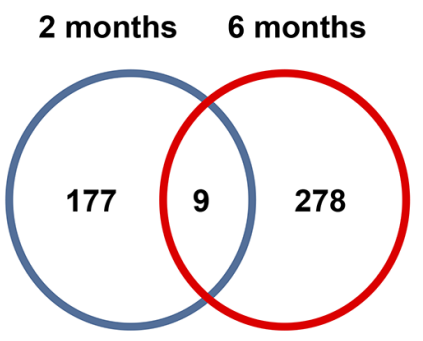

FIGURE 2 | Dual-omic approach to characterize the OB molecular homeostasis between 2-month-old and 6-month-old Tg2576 mice. (A) An overview of the experimental workflow used in this study. (B) Differential molecular profiling detected by-omics in Tg2576 OBs (2 and 6 months of age). The OB protein expression levels of amyloid precursor protein (APP) at early Alzheimer's disease (AD) stages in Tg2576 mice is shown. (C) Heat maps representing the degree of change for the differentially expressed proteins (Supplementary Table S2) between 2-and 6-month-old Tg2576 mice respect to wild-type (WT) littermates. Red and green, up- and down-regulated proteins, respectively. (D) Venn diagram for the differentially expressed genes detected in Tg2576 mice at both time points (Supplementary Table S1).

that prohibitin complex (constituted by Phb1 and Phb2) is a mitochondrial inner membrane-bound chaperone that participates in the mitochondrial respiratory complex assembly, modulates mitochondrial dynamics, and exerts beneficial effects on neurons by reducing free radical production (Artal-Sanz and Tavernarakis, 2009; Zhou et al., 2012). Subsequent experiments were performed to monitor the expression of both Phb subunits in the $\mathrm{OB}$ from $\mathrm{Tg} 2576$ mice. As shown in Figure 5C, a significant drop in Phb1 levels was evidenced in 2-month-old Tg2576 OBs, increasing its levels at 6 months of age with respect to WT littermates. Interestingly, a similar trend was also observed for the Phb2 protein in Tg2576 mice (Figure 5D). OB Phb complex was also independently evaluated in WT and Tg2576 mice during aging. With respect to data obtained at
2 months of age, Phb1 and Phb2 protein levels are decreased at 6 and 18 months in WT animals, maintaining constant levels in Tg2576 mice during the aging process (Figures 5C,D). These data indicate that Phb complex is an early target of human mutated APP, suggesting that the stable maintenance of Phb levels may help to counteract the oxidative stress present in olfactory neurons during AD progression in Tg2576 mice.

\section{Human Mutated APP Modifies the Olfactory Signaling Routes in a Stage-Dependent Manner}

The molecular network analysis also pointed out functional links between APP and a cluster of survival kinases, such 


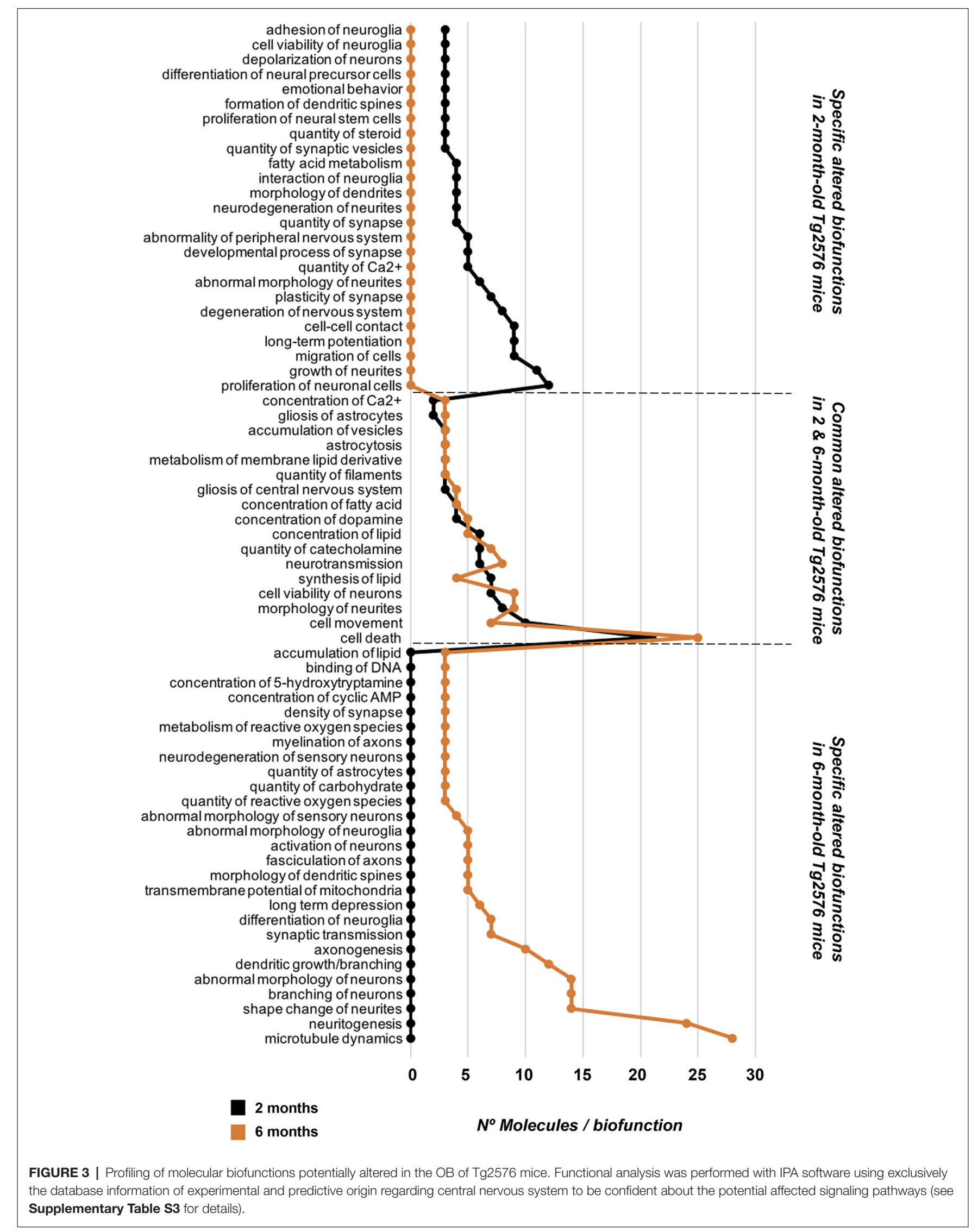




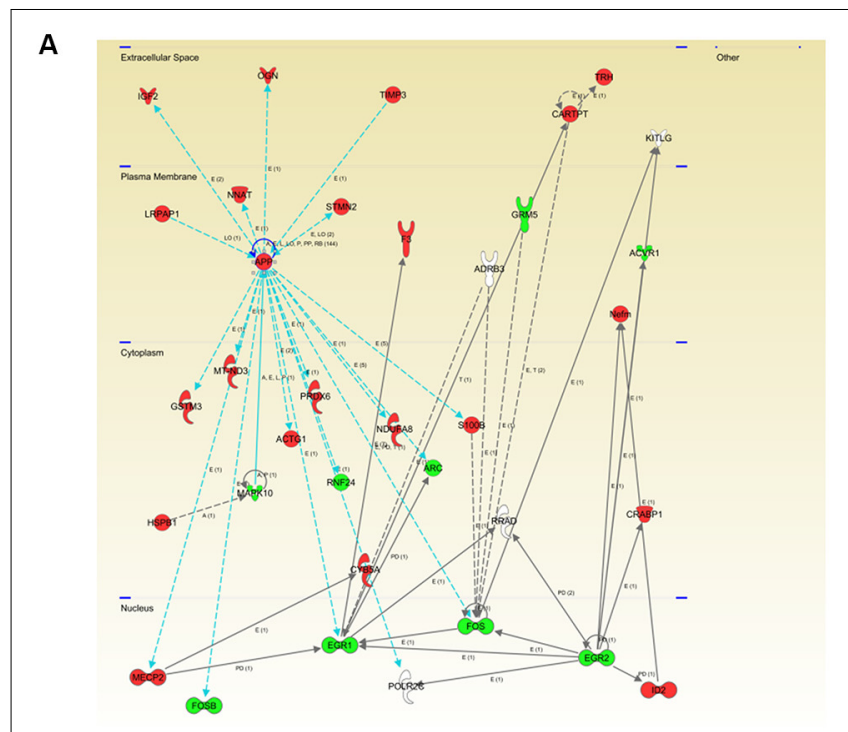

B

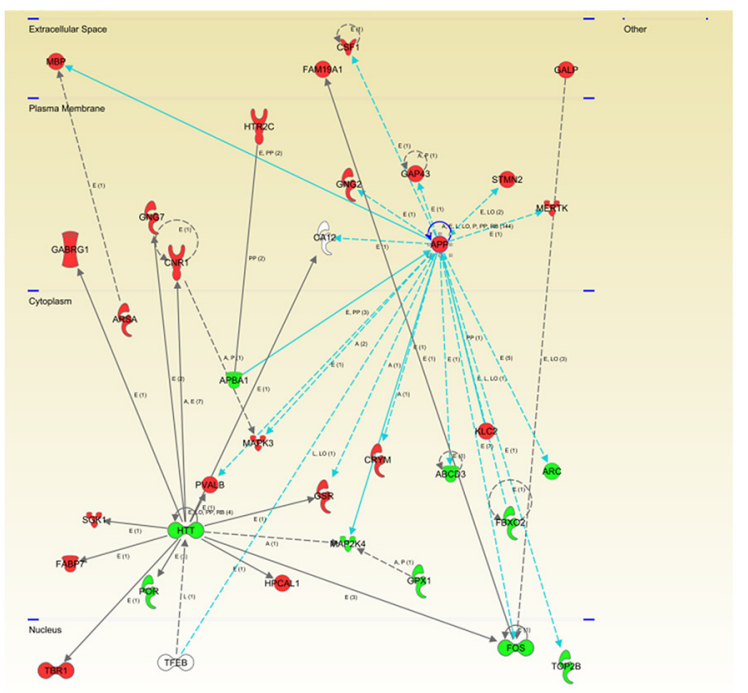

FIGURE 4 | Modulation of the APP functional interactome in Tg2576 mice at the level of OB. Adaptation of APP functional network in Tg2576 OBs at 2 months of age (A) and 6 months of age (B). Relationships between differential expressed genes/proteins and APP functional interactors are represented with blue lines. Continuous lines represent direct interactions, while discontinuous lines correspond to indirect functional interactions. Up-regulated molecules in red, and down-regulated molecules in green (See complete legend at: http://qiagen.force.com/KnowledgeBase/ KnowledgeNavigatorPage\#).

as ERK, p38 mitogen-activated protein kinase (MAPK), and Akt (Supplementary Figure S3). Subsequent experiments were performed to analyze the activation state of MAPKs, Akt, and p38 MAPK at pre-plaque stages in the $\mathrm{OB}$ of Tg2576 mice. A downstream inactivation in the MAPK pathway at the level of ERK was specifically observed in 2-monthold Tg2576 mice (Figure 6B), being the activation state of upstream MEK unaffected (Figure 6A). Moreover, olfactory Akt was specifically activated in 6-month-old transgenic mice (Figure 6C). In addition, Western-blot analysis revealed an increase in the activation status of OB p38 MAPK in 2-month-old transgenic mice (Figure 6D). This early activation was accompanied by a paralleled increment in the phosphorylation status of ATF2 (Figure 6D), a well-known downstream substrate of p38 MAPK (Puig et al., 2004). To complement our signaling mapping, other stress-responsive kinases were checked. The signal transduction of the SEK1stress-activated protein kinase (SAPK) axis was specifically activated in 6-month-old Tg2576 OBs (Figures 7A,B), while no appreciable changes were detected in the activation status of PKA (Figure 7C) and other survival kinases such as FAK, and PDK1/PKC axis with respect to WT animals (Supplementary Figure S2).

To deepen our understanding of the APP-dependent regulatory effects on kinase dynamics during the aging process in the OB of Tg2576 mice, steady-state levels and phosphorylated isoforms were independently evaluated in WT and Tg2576 mice during aging. For that, protein profiles were quantified in a time-dependent manner at 2-, 6-, and 18-months old (Figure 8). With respect to data obtained at 2 months of age, the activation of downstream ERK, and PDK1/PKC axis were constant in Tg2576 OBs, whereas a drop in the activation status of OB p38 MAPK was observed in 18-month-old Tg2576 mice when $\mathrm{AD}$ pathology is well established. No changes were observed in the activation state of olfactory SEK1 during the aging process in $\mathrm{Tg} 2576$ mice, in contrast with the inactivation observed in 6-, and 18-month-old WT animals (Figure 8). Conversely, a progressive inactivation was detected in its kinase downstream cascade as evidenced by SAPK dephosphorylation in Tg2576 mice (6-, and 18-month old). In addition, a significant variation was also observed in OB PKA levels in 6-month-old Tg2576.

\section{Olfactory SEK1/MKK4 and PKA Signaling Are De-regulated Across Human AD Grading}

Much effort has been spent on studying the role of $A \beta$ in sporadic Alzheimer's disease (sAD) pathogenesis but the available information is insufficient to fully understand the disease progression at the level of olfactory signaling (LachénMontes et al., 2017). To investigate whether SEK1 and PKA signaling pathways perturbed in the $\mathrm{OB}$ of $\mathrm{Tg} 2576$ mice were also associated with human $\mathrm{sAD}$, the activation state of the corresponding survival pathways was measured by Western blotting in OBs from sAD subjects with different neuropathological grading (Table 1). First, we performed immunohistochemical analysis to localize SEK1 and PKA in human OB. As shown in Supplementary Figures S4A,B, positive staining for the activated form of SEK1 was observed in the $\mathrm{OB}$ astrocytes and neurons. However, a specific mild staining for non-phosphorylated SEK1 was observed in neurons (Supplementary Figures S4C,D). With respect to an activated form of PKA, a diffuse staining of neuropil and all OB cellular components was observed (Supplementary Figures S4E,F), even in glial cells from granular layer (Supplementary Figure S4F), 


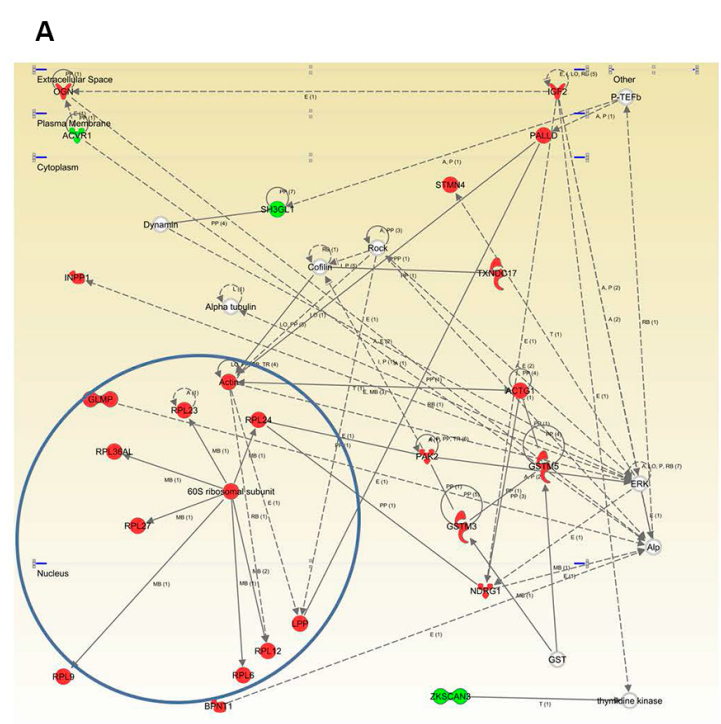

B
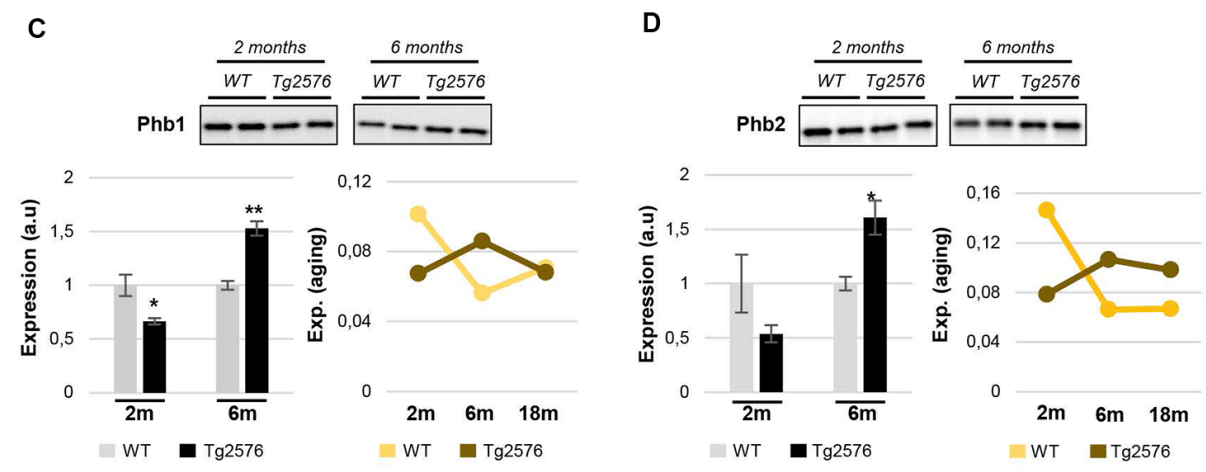

FIGURE 5 | Protein synthesis, and mitochondrial homeostasis are early compromised in Tg2576 at olfactory level. (A) Molecular network representing the up-regulation of ribosomal proteins (blue circles) in the OB of 2-month-old Tg2576. (B) Molecular network highlighting the dysregulation of specific components of the mitochondrial respiratory chain (Complex I, and Complex VI subunits) in the OB of 2-month-old Tg2576. (C) Time-dependent disruption of the olfactory Phb1 in Tg2576 mice. Phb expression was monitored by Western-blotting. (D) Time-dependent disruption of the olfactory Phb2 in Tg2576 mice. Phb expression was monitored by Western-blotting. Equal loading of the gels was assessed by stain-free digitalization. Panels show histograms of band densities. Data are presented as mean \pm standard error of the mean (SEM) from three independent OB samples per group $\left({ }^{*} P<0.05\right.$ vs. control group; ${ }^{* *} P<0.01$ vs. control group). Right graphs represent the expression of both Phb subunits during the aging process in WT and Tg2576 mice (2-, 6-, and 18-month-old).

while a mild staining in neuropil was observed for the non-phosphorylated PKA (Supplementary Figures S4G,H). As shown in Figure 9, the activation of SEK1 was specifically increased in advanced AD stages (Braak V-VI; Figure 9A). However, PKA activity was significantly increased in the initial (Braak I-II) AD stage, with respect to subjects with normal neuropathological examination (Figure 9B). In intermediate AD stage (Braak III-IV), a significant increment was also observed in total and activated PKA levels (Figure 9B).

\section{DISCUSSION}

We consider that a better understanding of the molecular events affected by the progressive accumulation of amyloid pathology might offer new olfactory targets for earlier diagnosis and therapeutic intervention. In particular, we report that: (i) multiple $\mathrm{OB}$ proteotranscriptomic variations appear at pre-plaque stages in Tg2576 mice; (ii) the functional interactome of the hAPPSw isoform at olfactory level is progressively modulated in these mice; (iii) the mitochondrial PHB complex was compromised at early stages in Tg2576 OBs; (iv) specific olfactory signaling routes (Akt, p38 MAPK, SEK1, and SAPK) were modulated in a time-dependent manner respect to WT animals; (v) APP overproduction induced specific regulatory effects on kinase dynamics (SEK1/SAPK, PKA) during the aging process in Tg2576 mice; and (vi) the SEK1 and PKA pathways were differentially activated during human AD grading at the level of the OB. All these findings at the very early pre-plaque stage provided mechanistic clues on the olfactory mechanisms involved in the progression of cognitive deficits previously reported in these mice (Cuadrado-Tejedor and García-Osta, 2014). 


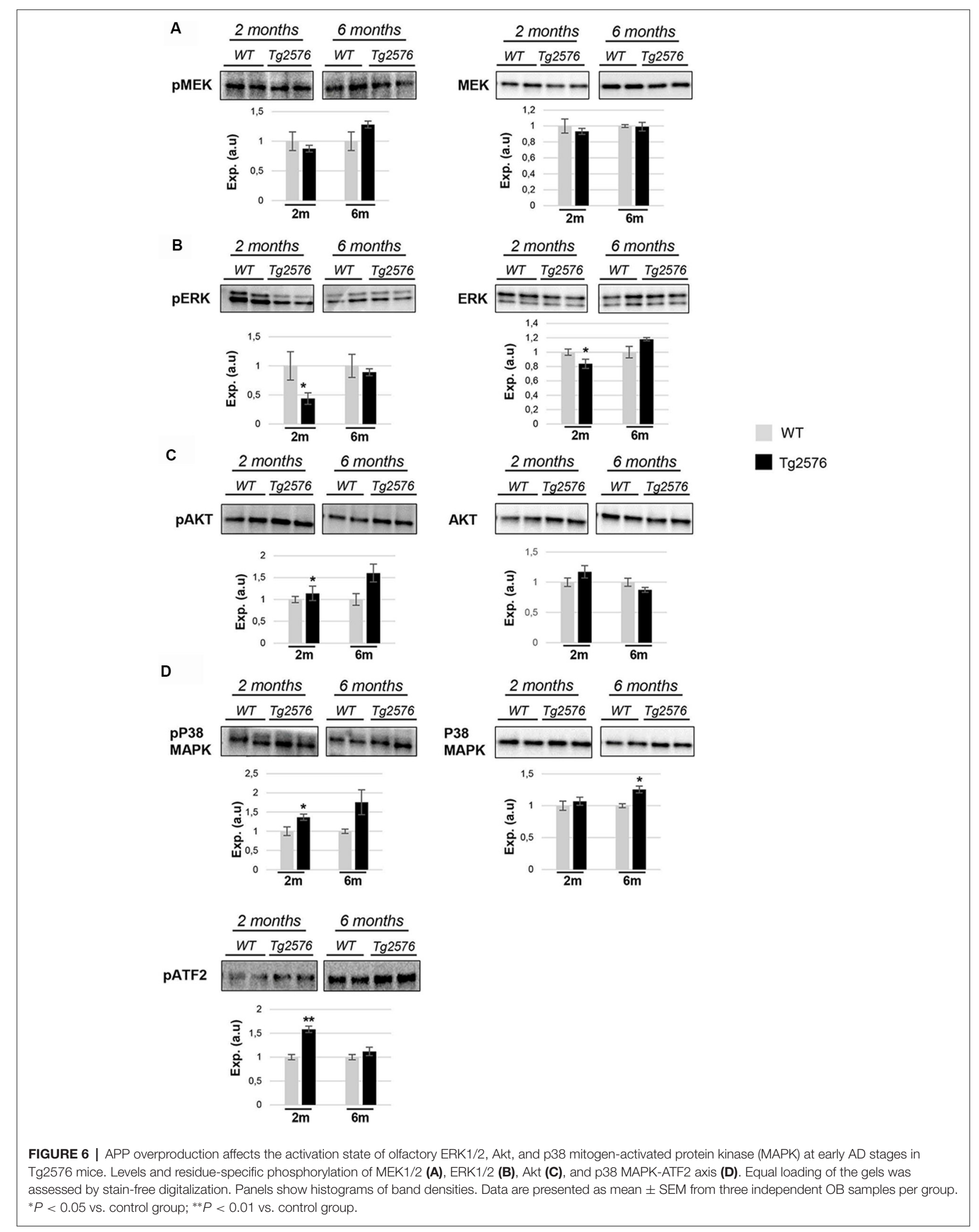



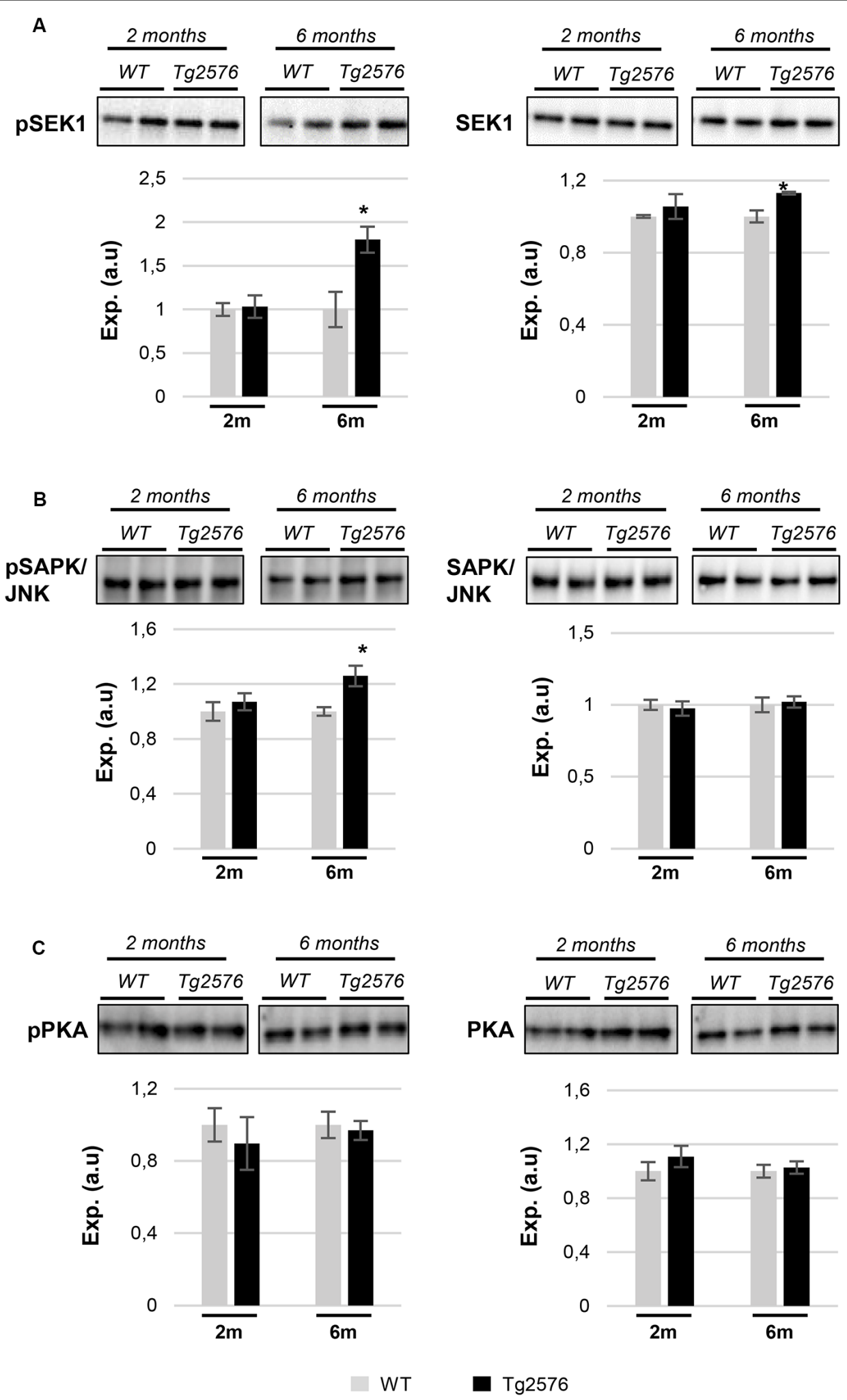

FIGURE 7 | APP overproduction specifically modulates the activation of the SEK1/MKK4-stress-activated protein kinase (SAPK)/JNK axis in 6-month-old Tg2576 mice. Time-dependent expression of total and phosphorylated levels of SEK1 (A), SAPK/JNK (B), and protein kinase A (PKA; C). Equal loading of the gels was assessed by stain-free digitalization. Panels show histograms of band densities. Data are presented as mean \pm SEM from three independent OB samples per group. ${ }^{*} P<0.05$ vs. control group. 


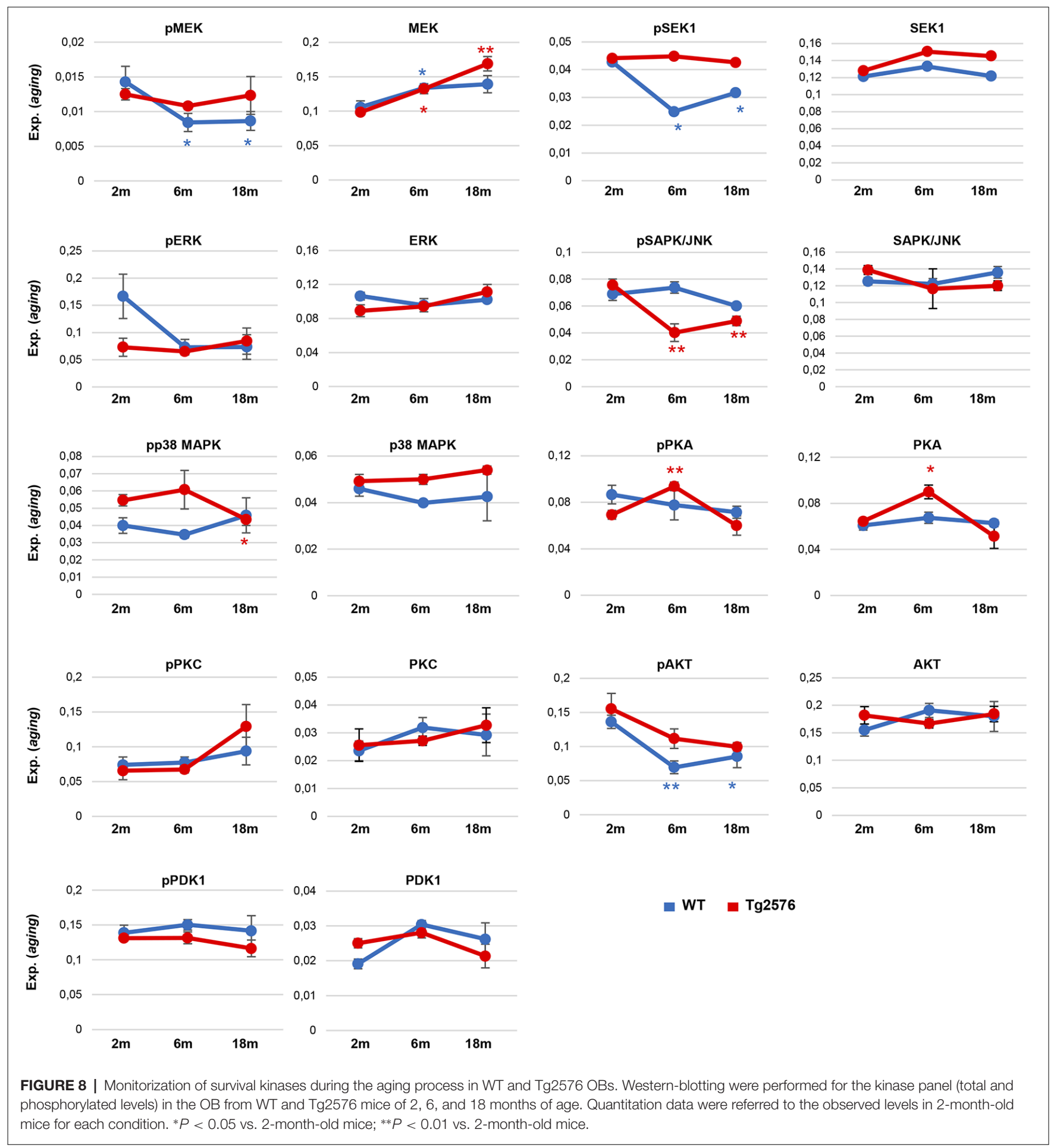

To systematically assess the global effect of olfactory APP production on gene expression at the transcript and protein level, we used a combinatorial omics analysis. The minimal overlap observed in transcriptome and proteome remodelings between 2-, and 6-month-old Tg2576 mice support the hypothesis that distinctive pathophysiological processes are involved in the $\mathrm{OB}$ during the initial progression of AD-like amyloid pathology.
For example, functional clustering suggested that changes in the growth of neurites (in 2-month-old transgenic mice), and microtubule dynamics in the OB (in 6-month-old Tg2576 mice) occur in the absence of neuropathological amyloid plaques, supporting the notion that cytoskeletal remodeling is an early AD pathological hallmark (Do Carmo et al., 2018). Despite the experimental and technical noise in both mRNA and protein 


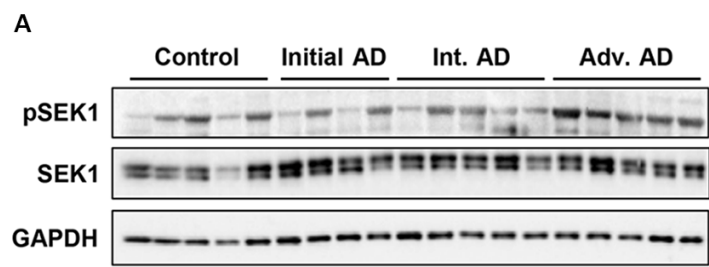

B

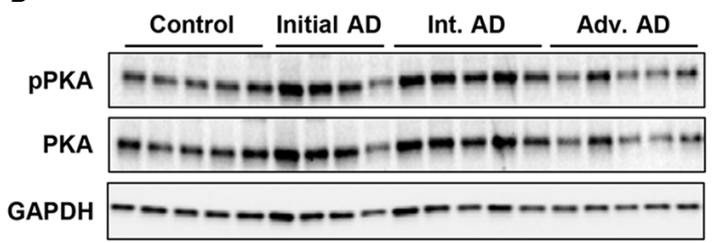

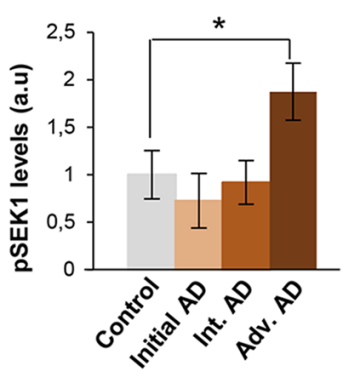
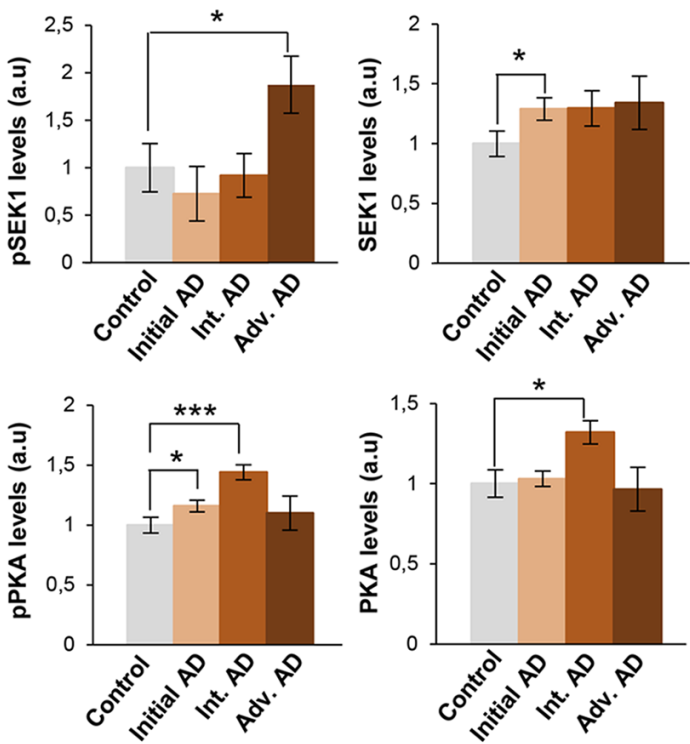

FIGURE 9 | Olfactory SEK1/MKK4 and PKA are differentially activated across Braak stages in human AD. Levels and residue-specific phosphorylation of SEK1/MKK4 (A), and PKA (B) in the OB across AD phenotypes. Equal loading of the gels was assessed by Ponceau staining and hybridization with a GAPDH specific antibody. Right panels show histograms of band densities. Data are presented as mean \pm SEM from five independent OB samples per group. ${ }^{*} P<0.05$ vs. control group; ${ }^{* * *} P<0.001$ vs. control group. Representative Western blot gels are shown.

measurements, which may contribute to an underestimation of true correlations, RNA-protein correlation was missing in our study. Buffering of mRNA variation against protein levels can occur at multiple levels, including intra- and interindividual genomic variation (Battle et al., 2015; Liu et al., 2016). The discrepancy we observed may be due to: (i) the use of different set of animals for each technological platform; (ii) the spatial and temporal delayed synthesis between mRNA and protein (Liu et al., 2016); (iii) post-transcriptional events; and (iv) the different hydrophobicity and solubility of specific proteome subsets (e.g., olfactory receptors), which hampers their characterization and quantitation by mass-spectrometry.

Prohibitin deficiencies have been previously associated with neurodegenerative phenotypes (Merkwirth et al., 2012; Dutta et al., 2018). During aging, our data indicated that Phb levels are stable while mutated APP is overproduced, probably to counteract the disease-aggravating oxidative stress during $\mathrm{AD}$ progression in Tg2576 mice. Generally, repression/induction of Phb1 is paralleled by a concomitant decrease/increase of its assembly partner Phb2 (Sánchez-Quiles et al., 2010; Merkwirth et al., 2012). Accordingly, OB Phb subunits are functionally interdependent in Tg2576 mice. In contrast, Phb subunits are not interdependent in the $\mathrm{OB}$ during $\mathrm{AD}$ neurodegeneration in humans (Lachén-Montes et al., 2017), indicating that the tangled regulatory mechanisms that govern the mitochondrial homeostasis in olfactory cells significantly differ between transgenic mice and sporadic human AD. Aberrant regulation of a subset of kinases may represent the triggering events leading to the spread of a perturbed signaling in AD (Perluigi et al., 2016). p38 MAPK is a multifunctional kinase that is activated by $A \beta$ in cultured neurons (Criscuolo et al., 2015), phosphorylates Tau protein (Li et al., 2003; Ferrer et al., 2005), and mediates the A $\beta$-induced inflammatory activation (Bachstetter et al., 2011). Different alterations of p38 MAPK pathway have been observed in the $\mathrm{OB}$, hippocampus, and cortical areas at early stages in human AD (Hensley et al., 1999; Sun et al., 2003; Munoz and Ammit, 2010; Criscuolo et al., 2015). In Tg2576 mice, we observed an early activation of p38 MAPK (validated by paralleled increase in ATF2 phosphorylation levels), suggesting detrimental effects, such as neuroinflammation and excitotoxicity at the level of the OB (Yi et al., 2018). Although Akt has been recently proposed as a therapeutic target for $\mathrm{AD}$-associated memory impairments (Griffin et al., 2005), different results have been obtained about the Akt activation across brain structures of human AD (Rickle et al., 2004; Petersen et al., 2007; Lachén-Montes et al., 2016). The specific activation of olfactory Akt observed in 6-month-old Tg2576 mice indicates potential protective mechanisms against memory impairments and synaptic deficits (Griffin et al., 2005). In an effort to delineate the oxidative stress signaling events in the OB of Tg2576 mice, we observed an increment in the activation of SAPK/JNK pathway in 6-month-old Tg2576 OBs. This activation precedes the $A \beta$ deposition, although $A \beta$ may enhance its activation at a later time (Zhu et al., 2001). In the human brain, phospho-SAPK is significantly increased in AD over control cases, overlapping with Tau-positive neurofibrillary pathology (Bachstetter et al., 2011; Kelly, 2018). In this study, we have detected an increment in the expression of phosphorylated SEK1 (an upstream activator of the SAPK/JNK route) exclusively in subjects with advanced AD stage (Braak V-VI). Previous reports suggest that this activation may play a role in the tau phosphorylation, and consequently, the formation of NFTs in 
late $\mathrm{AD}$ stages (Zhu et al., 2001). PKA is a tau-kinase and its expression/activity tends to be reduced in different contexts of AD pathology (Liang et al., 2007). In humans, a decrease in PKA activity was observed in the temporal cortex from AD subjects with a Braak stage V-VI (Iulita et al., 2014). However, we report a PKA overactivation that occurs in the $\mathrm{OB}$ derived from $\mathrm{AD}$ subjects with initial stages (Braak I-II), indicating that CAMP signaling appears to be stage and brain region specific.

Although our study has uncovered many intricacies in $\mathrm{OB}$ molecular homeostasis during early stages of $\mathrm{AD}$-related amyloidogenic pathology, there are potential limitations of our study that warrant discussion. First, due to technological issues, we failed to accurately quantify many proteins expressed at low levels that might also participate in the olfactory $\mathrm{AD}$ progression in Tg2576 mice. Second, our results are limited by transcript/protein abundance averaging among the multiple cell layers present in the $\mathrm{OB}$, hampering the exploration of olfactory cell-type specific molecular alterations. Third, A $\beta, A P P$, and its derived species may co-exist inside neurons (Crowe et al., 2018), and based on our experimental workflow, we cannot pinpoint which APP-derived species are responsible for the observed molecular disturbances. Finally, this study should be complemented with behavior testing to characterize which molecular abnormalities are directly linked to the cognitive deficit, as well as additional omic studies performed in different brain areas to verify the specificity of the molecular alterations detected at the level of the $\mathrm{OB}$.

\section{CONCLUSION}

Our dual-omic approach revealed the disruption of multiple molecular pathways at early stages of the OB amyloid pathology, leading to the identification of differential olfactory targets linked to APP metabolism. These findings point out the potential utility of alternative olfactory pathways for disease modification, in a stage-dependent manner, through intranasal therapies (Cheng et al., 2017) based on enzyme replacement or specific drug delivery (Agrawal et al., 2018).

\section{ETHICS STATEMENT}

\section{Human Samples}

According to the Spanish Law 14/2007 of Biomedical Research, inform written consent forms of Brain Bank of IDIBELL, and Neurological Tissue Bank of IDIBAPS-Hospital Clinic (Barcelona, Spain) was obtained for research purposes from relatives of patients included in this study. The study was conducted in accordance with the Declaration of Helsinki and all assessments, post-mortem evaluations, and procedures were previously approved by the Clinical Ethics Committee of Navarra Health Service.

\section{Animals}

All animal care and experimental procedures were in accordance with European and Spanish regulations (86/609/CEE;
RD1201/2005) and were approved by the Ethical Committee of the University of Navarra (no. 018/05).

\section{AUTHOR CONTRIBUTIONS}

JF-I and ES designed and supervised the complete study. ML-M, AG-M, MP and KA performed sample preparation, transcriptomic experiments, and functional assays. JF-I performed liquid chromatography-tandem mass spectrometry analysis. MG-O, MZ, IF and AP-M performed immunohistochemical analysis and neuropathological characterizations. ES performed bioinformatics, network biology analysis, data interpretation and wrote the article.

\section{FUNDING}

This work was funded by grants from the Spanish Ministry of Economy and Competitiveness (Ministerio de Economía, Industria y Competitividad, Gobierno de España, MINECO; Ref. SAF2014-59340-R), Department of Economic Development from Government of Navarra (Ref. PC023-PC024, PC025, PC081-82, PI59 and PC107-108) and Obra Social la Caixa to ES. AG-M and KA were supported by PEJ-2014-A-61949 and PEJ-2014-A-72151 (MINECO). ML-M and AG-M are supported by a predoctoral fellowship from the Public University of Navarra (UPNA). The Proteomics Unit of Navarrabiomed is a member of Proteored, PRB3-ISCIII, and is supported by grant PT17/0019/009, of the PE I + D + I 2013-2016 funded by ISCIII and FEDER.

\section{ACKNOWLEDGMENTS}

We are very grateful to the patients and relatives who generously donated the brain tissue for research purposes. We are indebted to the Neurological Tissue Banks of IDIBELL and IDIBAPS-Hospital Clinic (Barcelona, Spain), for sample and data procurement. The Clinical Neuroproteomics group of Navarrabiomed is a member of the Spanish Network of Olfaction (ROE). This project is part of the HUPO Brain Proteome Project and these results are lined up with the Spanish Initiative on the Human Proteome Project (SpHPP).

\section{SUPPLEMENTARY MATERIAL}

The Supplementary Material for this article can be found online at: https://www.frontiersin.org/articles/10.3389/fnagi. 2019.00141/full\#supplementary-material

FIGURE S1 | Olfactory $\beta$-Amyloid pathology increases with the disease progression in TG2576 mice. OBs were harvested from 2 (A-C), 6 (D-F) and 14 (G-J) month-old Tg2576 mice. Intraneuronal A $\beta$ immunoreactivity can be observed in 2 month-old mice (arrow heads; panel $\mathbf{B}$ and more detailed in panel $\mathbf{C}$ ). OB samples from 6 month-old animals (panels $\mathbf{D , E}$ and $\mathbf{F}$ ) shows moderate $A \beta$ deposition in form of diffuse plaques (asterisk). By contrast, mature plaques (asterisk in panel $\mathrm{G}, \mathrm{H}$ and insert $\mathrm{J}$ ) and vascular $\mathrm{A} \beta$ (insert $\mathrm{I}$ ) is evident in 14 month-old Tg2576. Scale bars $500 \mu \mathrm{m}$ for panels A, D, G, $100 \mu \mathrm{m}$ for panels $\mathbf{B}, \mathbf{E}, \mathbf{H}$ or $10 \mu \mathrm{m}$ (C, F, I, J). (GL: glomerular layer; EPL: external plexiform layer; MC: mitral layer; GrL: granular layer; AON: anterior olfactory nucleus). 
FIGURE S2 | OB PP5 up-regulation in 6-month-old Tg2576 mice $(* * P<0.01$ respect to control group) (A). No significant changes were found in FAK and PDK1/PKC signaling axis (B).

FIGURE S3 | Visualization of predictive interactions between APP and survival kinases (orange circles) based on differential datasets derived from 2-month-old Tg2576 mice.

FIGURE S4 | Immunohistochemical localization of OB SEK1 and PKA (phosphorylated and non-phosphorylated forms). Representative

\section{REFERENCES}

Agrawal, M., Saraf, S., Saraf, S., Antimisiaris, S. G., Chougule, M. B., Shoyele, S. A., et al. (2018). Nose-to-brain drug delivery: an update on clinical challenges and progress towards approval of anti-Alzheimer drugs. J. Control. Release 281, 139-177. doi: 10.1016/j.jconrel.2018.05.011

Alafuzoff, I., Arzberger, T., Al-Sarraj, S., Bodi, I., Bogdanovic, N., Braak, H., et al. (2008). Staging of neurofibrillary pathology in Alzheimer's disease: a study of the BrainNet Europe Consortium. Brain Pathol. 18, 484-496. doi: 10.1111/j. 1750-3639.2008.00147.x

Artal-Sanz, M., and Tavernarakis, N. (2009). Prohibitin and mitochondrial biology. Trends Endocrinol. Metab. 20, 394-401. doi: 10.1016/j.tem.2009. 04.004

Attems, J., Walker, L., and Jellinger, K. A. (2014). Olfactory bulb involvement in neurodegenerative diseases. Acta Neuropathol. 127, 459-475. doi: 10.1007/s00401-014-1261-7

Bachstetter, A. D., Xing, B., de Almeida, L., Dimayuga, E. R., Watterson, D. M., and Van Eldik, L. J. (2011). Microglial p38 $\alpha$ MAPK is a key regulator of proinflammatory cytokine up-regulation induced by toll-like receptor (TLR) ligands or $\beta$-amyloid $(\mathrm{A} \beta)$. J. Neuroinflammation 8:79. doi: 10.1186/17422094-8-79

Battle, A., Khan, Z., Wang, S. H., Mitrano, A., Ford, M. J., Pritchard, J. K., et al. (2015). Genomic variation. Impact of regulatory variation from RNA to protein. Science 347, 664-667. doi: 10.1126/science.1260793

Braak, H., Alafuzoff, I., Arzberger, T., Kretzschmar, H., and Del Tredici, K. (2006). Staging of Alzheimer disease-associated neurofibrillary pathology using paraffin sections and immunocytochemistry. Acta Neuropathol. 112, 389-404. doi: 10.1007/s00401-006-0127-z

Chen, G., Chen, K. S., Knox, J., Inglis, J., Bernard, A., Martin, S. J., et al. (2000). A learning deficit related to age and $\beta$-amyloid plaques in a mouse model of Alzheimer's disease. Nature 408, 975-979. doi: 10.1038/350 50103

Cheng, Y. S., Chen, Z. T., Liao, T. Y., Lin, C., Shen, H. C., Wang, Y. H., et al. (2017). An intranasally delivered peptide drug ameliorates cognitive decline in Alzheimer transgenic mice. EMBO Mol. Med. 9, 703-715. doi: 10.15252/emmm.201606666

Criscuolo, C., Fabiani, C., Bonadonna, C., Origlia, N., and Domenici, L. (2015). BDNF prevents amyloid-dependent impairment of LTP in the entorhinal cortex by attenuating p38 MAPK phosphorylation. Neurobiol. Aging 36, 1303-1309. doi: 10.1016/j.neurobiolaging.2014.11.016

Crowe, T. P., Greenlee, M. H. W., Kanthasamy, A. G., and Hsu, W. H. (2018). Mechanism of intranasal drug delivery directly to the brain. Life Sci. 195, 44-52. doi: $10.1016 /$ j.lfs.2017.12.025

Cuadrado-Tejedor, M., Garcia-Barroso, C., Sanchez-Arias, J. A., Rabal, O., PerezGonzalez, M., Mederos, S., et al. (2016). A first-in-class small-molecule that acts as a dual inhibitor of hdac and pde5 and that rescues hippocampal synaptic impairment in Alzheimer's disease mice. Neuropsychopharmacology 42, 524-539. doi: 10.1038/npp.2016.163

Cuadrado-Tejedor, M., and García-Osta, A. (2014). Current animal models of Alzheimer's disease: challenges in translational research. Front. Neurol. 5:182. doi: 10.3389/fneur.2014.00182

Daulatzai, M. A. (2015). Olfactory dysfunction: its early temporal relationship and neural correlates in the pathogenesis of Alzheimer's disease. J. Neural Transm. 122, 1475-1497. doi: 10.1007/s00702-015-1404-6

Do Carmo, S., Crynen, G., Paradis, T., Reed, J., Iulita, M. F., Ducatenzeiler, A., et al. (2018). Hippocampal proteomic analysis reveals distinct pathway deregulation profiles at early and late stages in a rat model of Alzheimer's-like immunohistochemical staining pattern of pSEK1 (A,B), SEK1 (C,D), pPKA (E,F) and PKA $(\mathbf{G}, \mathbf{H})$ in control and $A D$ cases.

TABLE S1 | Differential OB transcriptome in 2- and 6-month-old Tg2576 mice respect to WT mice.

TABLE S2 | Diffefrential OB proteome in 2- and 6-month-old Th2576 mice respect to WT mice.

TABLE S3 | Functional analysis of omics datasets.

amyloid pathology. Mol. Neurobiol. 55, 3451-3476. doi: 10.1007/s12035-0170580-9

Dutta, D., Ali, N., Banerjee, E., Singh, R., Naskar, A., Paidi, R. K., et al. (2018). Low levels of prohibitin in substantia nigra makes dopaminergic neurons vulnerable in Parkinson's disease. Mol. Neurobiol. 55, 804-821. doi: 10.1007/s12035-0160328-y

Ferrer, I., Gomez-Isla, T., Puig, B., Freixes, M., Ribe, E., Dalfo, E., et al. (2005). Current advances on different kinases involved in tau phosphorylation and implications in Alzheimer's disease and tauopathies. Curr. Alzheimer Res. 2, 3-18. doi: 10.2174/1567205052772713

Griffin, R. J., Moloney, A., Kelliher, M., Johnston, J. A., Ravid, R., Dockery, P., et al. (2005). Activation of Akt/PKB, increased phosphorylation of Akt substrates and loss and altered distribution of Akt and PTEN are features of Alzheimer's disease pathology. J. Neurochem. 93, 105-117. doi: 10.1111/j.1471-4159.2004. 02949.x

Guérin, D., Sacquet, J., Mandairon, N., Jourdan, F., and Didier, A. (2009). Early locus coeruleus degeneration and olfactory dysfunctions in $\operatorname{Tg} 2576$ mice. Neurobiol. Aging 30, 272-283. doi: 10.1016/j.neurobiolaging.2007.05.020

Hensley, K., Floyd, R. A., Zheng, N. Y., Nael, R., Robinson, K. A., Nguyen, X., et al. (1999). p38 kinase is activated in the Alzheimer's disease brain. J. Neurochem. 72, 2053-2058. doi: 10.1046/j.1471-4159.1999.0722053.x

Hsiao, K., Chapman, P., Nilsen, S., Eckman, C., Harigaya, Y., Younkin, S., et al. (1996). Correlative memory deficits, A $\beta$ elevation, and amyloid plaques in transgenic mice. Science 274, 99-102. doi: 10.1126/science.274. 5284.99

Irizarry, R. A., Bolstad, B. M., Collin, F., Cope, L. M., Hobbs, B., and Speed, T. P. (2003). Summaries of Affymetrix GeneChip probe level data. Nucleic Acids Res. 31:e15. doi: 10.1093/nar/gng015

Iulita, M. F., Allard, S., Richter, L., Munter, L. M., Ducatenzeiler, A., Weise, C., et al. (2014). Intracellular $A \beta$ pathology and early cognitive impairments in a transgenic rat overexpressing human amyloid precursor protein: a multidimensional study. Acta Neuropathol. Commun. 2:61. doi: 10.1186/20515960-2-61

Jacobsen, J. S., Wu, C. C., Redwine, J. M., Comery, T. A., Arias, R., Bowlby, M., et al. (2006). Early-onset behavioral and synaptic deficits in a mouse model of Alzheimer's disease. Proc. Natl. Acad. Sci. U S A 103, 5161-5166. doi: 10.1073/pnas.0600948103

Janus, C., Pearson, J., McLaurin, J., Mathews, P. M., Jiang, Y., Schmidt, S. D., et al. (2000). A $\beta$ peptide immunization reduces behavioural impairment and plaques in a model of Alzheimer's disease. Nature 408, 979-982. doi: 10.1038/350 50110

Kelly, M. P. (2018). Cyclic nucleotide signaling changes associated with normal aging and age-related diseases of the brain. Cell Signal. 42, 281-291. doi: 10.1016/j.cellsig.2017.11.004

Lachén-Montes, M., González-Morales, A., de Morentin, X. M., PérezValderrama, E., Ausin, K., Zelaya, M. V., et al. (2016). An early dysregulation of FAK and MEK/ERK signaling pathways precedes the $\beta$ amyloid deposition in the olfactory bulb of APP/PS1 mouse model of Alzheimer's disease. J. Proteomics 148, 149-158. doi: 10.1016/j.jprot.2016. 07.032

Lachén-Montes, M., González-Morales, A., Zelaya, M. V., Pérez-Valderrama, E., Ausin, K., Ferrer, I., et al. (2017). Olfactory bulb neuroproteomics reveals a chronological perturbation of survival routes and a disruption of prohibitin complex during Alzheimer's disease progression. Sci. Rep. 7:9115. doi: 10.1038/s41598-017-09481-x

Lachen-Montes, M., Zelaya, M., Segura, V., Fernández-Irigoyen, J., and Santamaría, E. (2017). Progressive modulation of the human olfactory bulb 
transcriptome during Alzheimer's disease evolution: novel insights into the olfactory signaling across proteinopathies. Oncotarget 8, 69663-69679. doi: 10.18632/oncotarget.18193

Lehman, E. J., Kulnane, L. S., and Lamb, B. T. (2003). Alterations in $\beta$-amyloid production and deposition in brain regions of two transgenic models. Neurobiol. Aging 24, 645-653. doi: 10.1016/s0197-4580(02)00153-7

Li, Y., Liu, L., Barger, S. W., and Griffin, W. S. (2003). Interleukin-1 mediates pathological effects of microglia on tau phosphorylation and on synaptophysin synthesis in cortical neurons through a p38-MAPK pathway. J. Neurosci. 23, 1605-1611. doi: 10.1523/JNEUROSCI.23-05-01605.2003

Liang, Z., Liu, F., Grundke-Iqbal, I., Iqbal, K., and Gong, C. X. (2007). Downregulation of cAMP-dependent protein kinase by over-activated calpain in Alzheimer disease brain. J. Neurochem. 103, 2462-2470. doi: 10.1111/j.14714159.2007.04942.x

Liu, Y., Beyer, A., and Aebersold, R. (2016). On the dependency of cellular protein levels on mRNA abundance. Cell 165, 535-550. doi: 10.1016/j.cell.2016.03.014

Merkwirth, C., Martinelli, P., Korwitz, A., Morbin, M., Brönneke, H. S., Jordan, S. D., et al. (2012). Loss of prohibitin membrane scaffolds impairs mitochondrial architecture and leads to tau hyperphosphorylation and neurodegeneration. PLoS Genet. 8:e1003021. doi: 10.1371/journal.pgen. 1003021

Munoz, L., and Ammit, A. J. (2010). Targeting p38 MAPK pathway for the treatment of Alzheimer's disease. Neuropharmacology 58, 561-568. doi: 10.1016/j.neuropharm.2009.11.010

Palomino-Alonso, M., Lachén-Montes, M., González-Morales, A., Ausín, K., Pérez-Mediavilla, A., Fernández-Irigoyen, J., et al. (2017). Network-driven proteogenomics unveils an aging-related imbalance in the olfactory $\mathrm{I} \kappa \mathrm{B} \alpha-\mathrm{NF} \kappa \mathrm{B}$ p65 complex functionality in Tg2576 Alzheimer's disease mouse model. Int. J. Mol. Sci. 18:E2260. doi: 10.3390/ijms18112260

Perluigi, M., Barone, E., Di Domenico, F., and Butterfield, D. A. (2016). Aberrant protein phosphorylation in Alzheimer disease brain disturbs pro-survival and cell death pathways. Biochim. Biophys. Acta 1862, 1871-1882. doi: 10.1016/j. bbadis.2016.07.005

Petersen, R. B., Nunomura, A., Lee, H. G., Casadesus, G., Perry, G., Smith, M. A., et al. (2007). Signal transduction cascades associated with oxidative stress in Alzheimer's disease. J. Alzheimers Dis. 11, 143-152. doi: 10.3233/JAD-200711202

Puig, B., Vinals, F., and Ferrer, I. (2004). Active stress kinase p38 enhances and perpetuates abnormal tau phosphorylation and deposition in Pick's disease. Acta Neuropathol. 107, 185-189. doi: 10.1007/s00401-003-0793-Z

Puzzo, D., Gulisano, W., Palmeri, A., and Arancio, O. (2015). Rodent models for Alzheimer's disease drug discovery. Expert. Opin. Drug Discov. 10, 703-711. doi: 10.1517/17460441.2015.1041913

Reddy, P. H., McWeeney, S., Park, B. S., Manczak, M., Gutala, R. V., Partovi, D., et al. (2004). Gene expression profiles of transcripts in amyloid precursor protein transgenic mice: up-regulation of mitochondrial metabolism and apoptotic genes is an early cellular change in Alzheimer's disease. Hum. Mol. Genet. 13, 1225-1240. doi: 10.1093/hmg/ddh140

Rey, N. L., Wesson, D. W., and Brundin, P. (2018). The olfactory bulb as the entry site for prion-like propagation in neurodegenerative diseases. Neurobiol. Dis. 109, 226-248. doi: 10.1016/j.nbd.2016.12.013

Rickle, A., Bogdanovic, N., Volkman, I., Winblad, B., Ravid, R., and Cowburn, R. F. (2004). Akt activity in Alzheimer's disease and other neurodegenerative disorders. Neuroreport 15, 955-959. doi: 10.1097/00001756-20040429000005

Sanchez-Ortiz, E., Hahm, B. K., Armstrong, D. L., and Rossie, S. (2009). Protein phosphatase 5 protects neurons against amyloid- $\beta$ toxicity. J. Neurochem. 111, 391-402. doi: 10.1111/j.1471-4159.2009.06337.x

Sánchez-Quiles, V., Santamaria, E., Segura, V., Sesma, L., Prieto, J., and Corrales, F. J. (2010). Prohibitin deficiency blocks proliferation and induces apoptosis in human hepatoma cells: molecular mechanisms and functional implications. Proteomics 10, 1609-1620. doi: 10.1002/pmic.200900757

Sasaguri, H., Nilsson, P., Hashimoto, S., Nagata, K., Saito, T., De Strooper, B., et al. (2017). APP mouse models for Alzheimer's disease preclinical studies. EMBO J. 36, 2473-2487. doi: 10.15252/embj.201797397
Silva, A. R., Grinberg, L. T., Farfel, J. M., Diniz, B. S., Lima, L. A., Silva, P. J., et al. (2012). Transcriptional alterations related to neuropathology and clinical manifestation of Alzheimer's disease. PLoS One 7:e48751. doi: 10.1371/journal. pone. 0048751

Sun, A., Liu, M., Nguyen, X. V., and Bing, G. (2003). P38 MAP kinase is activated at early stages in Alzheimer's disease brain. Exp. Neurol. 183, 394-405. doi: 10.1016/s0014-4886(03)00180-8

Tyanova, S., Temu, T., and Cox, J. (2016a). The MaxQuant computational platform for mass spectrometry-based shotgun proteomics. Nat. Protoc. 11, 2301-2319. doi: 10.1038/nprot.2016.136

Tyanova, S., Temu, T., Sinitcyn, P., Carlson, A., Hein, M. Y., Geiger, T., et al. (2016b). The Perseus computational platform for comprehensive analysis of (prote)omics data. Nat. Methods 13, 731-740. doi: 10.1038/nmeth. 3901

Vizcaíno, J. A., Deutsch, E. W., Wang, R., Csordas, A., Reisinger, F., Rios, D., et al. (2014). ProteomeXchange provides globally coordinated proteomics data submission and dissemination. Nat. Biotechnol. 32, 223-226. doi: 10.1038/ nbt. 2839

Wesson, D. W., Borkowski, A. H., Landreth, G. E., Nixon, R. A., Levy, E., and Wilson, D. A. (2011). Sensory network dysfunction, behavioral impairments and their reversibility in an Alzheimer's $\beta$-amyloidosis mouse model. J. Neurosci. 31, 15962-15971. doi: 10.1523/JNEUROSCI.2085 $-11.2011$

Wesson, D. W., Levy, E., Nixon, R. A., and Wilson, D. A. (2010). Olfactory dysfunction correlates with amyloid- $\beta$ burden in an Alzheimer's disease mouse model. J. Neurosci. 30, 505-514. doi: 10.1523/JNEUROSCI.4622 $-09.2010$

Westerman, M. A., Cooper-Blacketer, D., Mariash, A., Kotilinek, L., Kawarabayashi, T., Younkin, L. H., et al. (2002). The relationship between A $\beta$ and memory in the Tg2576 mouse model of Alzheimer's disease. J. Neurosci. 22, 1858-1867. doi: 10.1523/JNEUROSCI.22-05-01858.2002

Yi, J. H., Baek, S. J., Heo, S., Park, H. J., Kwon, H., Lee, S., et al. (2018). Direct pharmacological Akt activation rescues Alzheimer's disease like memory impairments and aberrant synaptic plasticity. Neuropharmacology 128, 282-292. doi: 10.1016/j.neuropharm.2017.10.028

Young, J. W., Sharkey, J., and Finlayson, K. (2009). Progressive impairment in olfactory working memory in a mouse model of Mild Cognitive Impairment. Neurobiol. Aging 30, 1430-1443. doi: 10.1016/j.neurobiolaging.2007. 11.018

Zelaya, M. V., Pérez-Valderrama, E., de Morentin, X. M., Tuñon, T., Ferrer, I., Luquin, M. R., et al. (2015). Olfactory bulb proteome dynamics during the progression of sporadic Alzheimer's disease: identification of common and distinct olfactory targets across Alzheimer-related co-pathologies. Oncotarget 6, 39437-39456. doi: 10.18632/oncotarget.6254

Zhou, P., Qian, L., D’Aurelio, M., Cho, S., Wang, G., Manfredi, G., et al. (2012). Prohibitin reduces mitochondrial free radical production and protects brain cells from different injury modalities. J. Neurosci. 32, 583-592. doi: 10.1523/JNEUROSCI.2849-11.2012

Zhu, X., Raina, A. K., Rottkamp, C. A., Aliev, G., Perry, G., Boux, H., et al. (2001). Activation and redistribution of c-jun $\mathrm{N}$-terminal kinase/stress activated protein kinase in degenerating neurons in Alzheimer's disease. J. Neurochem. 76, 435-441. doi: 10.1046/j.1471-4159.2001.00046.x

Conflict of Interest Statement: The authors declare that the research was conducted in the absence of any commercial or financial relationships that could be construed as a potential conflict of interest.

Copyright (C) 2019 Lachen-Montes, González-Morales, Palomino, Ausin, GómezOchoa, Zelaya, Ferrer, Pérez-Mediavilla, Fernández-Irigoyen and Santamaría. This is an open-access article distributed under the terms of the Creative Commons Attribution License (CC BY). The use, distribution or reproduction in other forums is permitted, provided the original author(s) and the copyright owner(s) are credited and that the original publication in this journal is cited, in accordance with accepted academic practice. No use, distribution or reproduction is permitted which does not comply with these terms. 